\title{
Modelling Enhancement of Cross-ventilation in Sheltered Buildings using Stochastic Optimization
}

\author{
Mohammadreza Shirzadi ${ }^{a}$, Parham A. Mirzaei ${ }^{\mathrm{b}, 1}$, Mohammad Naghashzadegan ${ }^{\mathrm{a}}$, Yoshihide \\ Tominaga ${ }^{\mathrm{c}}$ \\ ${ }^{a}$ Mechanical Engineering Department, University of Guilan, Rasht, Iran \\ ${ }^{\mathrm{b}}$ Architecture and Built Environment Department, University of Nottingham, Nottingham, UK \\ ${ }^{c}$ Department of Architecture and Building Engineering, Niigata Institute of Technology, 1719, Fujihashi, \\ Kashiwazaki, Japan
}

\begin{abstract}
Accurate representation of turbulence phenomenon in Computational Fluid Dynamics (CFD) modeling of cross-ventilation around and inside buildings is a challenging and complex problem, especially under the sheltering effect of surrounding buildings. Steady Reynolds Averaged NavierStokes (RANS) models are broadly used in many practical applications. However, these models mainly fail to predict accurate distribution of flow characteristics in the cavity formed between the buildings, and hence miscalculate the attributed cross flow and airflow rate through buildings. In this study, a novel and systematic methodology is proposed to enhance the accuracy of the $k-\varepsilon$ model for the urban study applications such as cross-ventilation in the sheltered buildings.

A microclimate CFD model for a case study of a cross-ventilation experimental work by Tominaga and Blocken [1] was firstly constructed and validated. In the next step, the closure coefficients of the $k-\varepsilon$ model were modified using a stochastic optimization and Monte Carlo Sampling techniques. The probability density function (PDF) of all closure coefficients were given to the optimizer and proper objective function defined in terms of different validation metrics. The modified coefficients obtained from the developed systematic method could successfully simulates the cross-ventilation phenomena inside the building with an airflow rate prediction error less than $8 \%$ compared to the experiment while other RANS models predicted the airflow rate with up to $70 \%$ error. The effectiveness of the optimization technique was also discussed in terms of validation metrics and pressure coefficients.
\end{abstract}

Keywords: CFD, Cross-ventilation, Turbulence, Optimization, Sheltered Building

\footnotetext{
${ }^{1}$ Corresponding author: University Park, Nottingham, NG2RD, UK

Tel.: +44 011595 14129; fax: +44 01159513159

Email: Parham.Mirzaei Ahranjani@nottingham.ac.uk
} 


\section{Nomenclature}

\begin{tabular}{|c|c|c|c|}
\hline$\rho$ & Density & $U_{H}$ & $\begin{array}{l}\text { Inflow mean streamwise velocity at building } \\
\text { height } H\end{array}$ \\
\hline$t$ & Time & $H$ & Building height \\
\hline \multirow[t]{2}{*}{$x_{i}$} & \multirow{2}{*}{$\begin{array}{l}\text { Three components of the spatial coordinate } \\
(i=1 \text {, streamwise }(x) ; i=2 \text {, lateral }(y) ; i= \\
3 \text {, vertical }(z))\end{array}$} & $\alpha$ & Power-law exponent \\
\hline & & $q$ & Hit rate \\
\hline \multirow[t]{2}{*}{$U_{i}$} & \multirow{2}{*}{$\begin{array}{l}\text { Three components of the mean velocity vector } \\
(i=1 \text {, streamwise; } i=2, \text { lateral; } i=3 \text {, } \\
\text { vertical })\end{array}$} & $N$ & Number of data points \\
\hline & & $O_{i}$ & Observed value \\
\hline$\tau_{i j}$ & Viscous stress tensor & $P_{i}$ & Predicted value \\
\hline$S_{M_{i}}$ & Momentum source & $F B$ & Fractional bias \\
\hline$\mu_{t}$ & Turbulent viscosity & $F A C 2$ & $\begin{array}{l}\text { Fraction of the predictions within a factor of } \\
2 \text { of the observations }\end{array}$ \\
\hline$\delta_{i j}$ & Kronecker Delta function & $N M S E$ & Normalized mean square error \\
\hline$k$ & Turbulent kinetic energy & $u_{i}$ & Three components of the fluctuating \\
\hline $\mathrm{P}$ & pressure & & $\begin{array}{l}\text { velocity vector }(i=1 \text {, streamwise; } i=2 \text {, } \\
\text { lateral; } i=3 \text {, vertical) }\end{array}$ \\
\hline$\mu_{e f f}$ & Effective viscosity & $\sigma_{k}$ & $k-\varepsilon$ model constant \\
\hline$C_{\mu}$ & $k-\varepsilon$ model constant & $\sigma_{\varepsilon}$ & $k-\varepsilon$ model constant \\
\hline$\mu$ & Molecular viscosity & $C_{\varepsilon 2}$ & $k-\varepsilon$ model constant \\
\hline$\varepsilon$ & Turbulent dissipation rate & $C_{\varepsilon 1}^{\varepsilon c}$ & $k-\varepsilon$ model constant \\
\hline$P_{k}$ & Shear production term & W & Building width \\
\hline $\mathrm{D}^{n}$ & Building length & $C_{p}$ & Pressure coefficient \\
\hline
\end{tabular}

\section{Introduction}

Buildings account for about $40 \%$ of total final energy consumption in the US and European countries [2]. Increasing rate of industrialization and urbanization, and emerging of more mega cities indicate the importance of integration of the energy saving strategies into the modern buildings [3]. Natural ventilation has been extensively used in traditional and modern buildings to improve thermal comfort and air quality [4, 5], and to decrease the cooling energy demand of buildings. Moreover air quality of dwellings and comfort level of occupants can be significantly enhanced by utilizing natural ventilation strategies [6]. Nonetheless, accurate design of buildings to benefit from these natural ventilation strategies highly depends on reliable prediction of the airflow parameters, crossing through buildings.

Different analytical and empirical models were developed in the last three decades to characterize natural ventilation in buildings. As descripted in [7], these methods include small-scale or full-scale experimental test, multi-zone and Computational Fluid Dynamic (CFD) models. In the recent years due to an exponential growth in the computational capability of the processors, application of the CFD models in wind-driven related topics such as cross-ventilation [8-10], energy prediction [11-15], pedestrian level ventilation [16, 17], and pollution dispersion [18, 19] has been noticeably surged. Most of the microclimate CFD studies adapted Reynolds-averaged Navier Stokes (RANS) as their turbulence models. The number of large eddy simulations (LES) [20-23] and unsteady Reynoldsaveraged Navier Stokes (URANS) [17,24] studies are considerably less due to the limitation of these models in terms of high computational cost and complexity. Therefore, steady RANS models are 
recognized as reliable and low cost simulation techniques, which are commonly accepted in the winddriven CFD studies.

In general, cross-ventilation researches can be classified into two categories of unsheltered or isolated (generic) building and sheltered scenarios where the effect of flow distraction caused by surrounding buildings or other environmental obstacles is also considered. Acceptable accuracy of CFD modeling of cross-ventilation for unsheltered scenario was reported in many researches. In the works presented by Ramponi and Blocken [8], Tepner et al. [9], van Hooff et al. [25], and Yusuf and Mirzaei [26] RANS and LES models were successfully applied to the unsheltered building scenario. Many studies also considered the sheltering effect of the neighborhood buildings on the airflow inside a building and around it at the pedestrian level [6, 17, 22, 27-34]. The complexity of the turbulent flow in the case of sheltered building was demonstrated in these studies while detailed representation of surrounding environment was emphasized. The complexity of the sheltering effects in CFD simulations is still a challenging issue for CFD users and it can be concluded that the accuracy of CFD models for sheltered buildings is still a challenging problem.

Due to the high complexity of URANS and LES models along with their inherent high computational cost, many of the above mentioned studies utilized RANS models for modeling of the turbulence. However, these models mainly fail to predict accurate distribution of the turbulent kinetic energy (TKE), specifically for the sheltered building and street canyon airflow scenarios. As described in [35-38], the RANS turbulence models are generally showing poor accuracy in the modeling of the flow separation over the roof and the wake region behind the buildings. This is mainly due to the inaccurate prediction of the momentum diffusion in the wake region inside the street canyon and behind the buildings. Also, the RANS turbulence modes are not inherently able to simulate the unsteady fluctuations around the building, which has a noticeable impact on the momentum diffusion in the wake region behind building [35]. This major limitation in the modeling of the TKE results in an inaccurate cross-ventilation pattern inside a sheltered building, which itself results in an inaccurate simulation of the airflow crossing the sheltered building. Despite some valuable guidelines for crossventilation modeling of unsheltered building using the RANS models, e.g. [8], a similar comprehensive study for the sheltered building scenario cannot be addressed. A few existing studies such as [22] are based on the highly expensive and complex LES models. Although some of the existing investigations utilized the RANS models in their study for cross-ventilation of generic buildings, e.g. [27, 39], the developed CFD models are mainly validated with the experimental data obtained from the unsheltered building scenarios.

Despite many modifications performed on the RANS turbulence models, e.g. $R N G k-\varepsilon$ [40] and Realizable $k-\varepsilon[41,42]$, their application for the atmospheric boundary layer (ABL) flow modeling is still a challenging issue [43]. In addition to the poor accuracy of these RANS models in predicting the momentum diffusion, there is another limitation related to the closure coefficients used in these models. This drawback is associated with the fact that the closure coefficients are mainly obtained based on different experimental and empirical data analysis based on some of the fundamental and classical flow problems, e.g. homogeneous decaying turbulence, free shear flow, and fully developed channel flow [44]. Nonetheless, such flows have limited similarities with the airflow characteristics in the ABL. Therefore, it can be concluded that there is an inherent uncertainty in these coefficients, and as demonstrated in $[44,45]$, the flow-independent values for these coefficients are unlikely to exist. 
Modification of the closure coefficients has been done previously in different CFD applications. Weihing et al. [46] investigated the turbulent heat transfer phenomenon in a heated channel with periodic surface ribs in which commonly used closure coefficients of RANS models fail to predict the massively separated flow behavior and turbulent heat flux. They showed the superiority of the full differential transport closures to the eddy-viscosity closures in capturing rapidly-evolving flow and thermal field. In another work by Shams et al. [47], the limitations of eddy-viscosity model for turbulent heat transfer prediction in low-Prandtl fluids were investigated while a set of new correlations and new closure coefficients were proposed for natural and mixed convection regimes using an algebraic heat flux model (AHFM) and the low-Reynolds $k-\varepsilon$ model. In the work presented by Duynkerke [48], the closure coefficients of the standard $k-\varepsilon$ model were modified based on a comparison with a LES simulation and a measurement study for neutral and stable ABL flows over a flat terrain. The value of the modified closure coefficients in this study were obtained to be $C_{\mu}=$ 0.033, $C_{\varepsilon 1}=1.46, C_{\varepsilon 2}=1.85, \sigma_{\varepsilon}=2.38$, and $\sigma_{k}=1$. A similar study by Detering and Etling [49] was done in which modified closure coefficients were presented for mesoscale ABL simulation above flat and complex terrain. In the recent years, thanks to the increased computational power, statistical techniques have been used by some researchers to calibrate the closure coefficients of the RANS models. In most of the statistical methods, e.g. [50, 51], the effect of the inherent uncertainty of the closure coefficients was investigated for different flow problems. Using the Latin Hypercube Sampling (LHS) method and different probability density function (PDF) forms for the closure coefficients of the standard $k-\varepsilon$ model, Dunn et al. [52] showed that the highest uncertainty of the flow parameters occurs in the recirculating region and near the reattachment point. Guillas et al. [53] proposed a Bayesian technique for calibration of the closure coefficients of the standard $k-\varepsilon$ model for a symmetric street canyon simulation. Their results showed that for $C_{\mu}$ and $C_{\varepsilon 2}$ values between $0.02 \leq C_{\mu} \leq 0.04$ and $2 \leq C_{\varepsilon 2} \leq 2.2$ the highest probability can be obtained to match the experimental data. Furthermore, in the work presented by Iqbal et al. [54], the modified closure coefficients proposed in [53] were used in a numerical analysis for the pedestrian wind environment around a group of high-rise buildings. They further altered the coefficients by performing two additional experiments and concluded that when $C_{\mu}=0.12, C_{\varepsilon 1}=1, \sigma_{k}=0.53, \sigma_{\varepsilon}=0.5$, and $C_{\varepsilon 2}=$ 1.92 better agreement with the experimental data can be achieved in comparison with the standard $k-$ $\varepsilon$ with the default coefficients.

According to literature, there is a lack of understanding in the impact of the closure coefficients on the predicted CFD results in urban studies. Hence, this study aims to develop a systematic and computationally cost effective method to optimize closure coefficients of the RANS turbulence models utilized in urban studies such as cross-ventilation through sheltered buildings. For this purpose, the microclimate CFD model of a sheltered building scenario is initially developed and validated using an experimental work by Tominaga and Blocken [1]. The accuracy of different turbulence models, i.e. the standard $k-\varepsilon$ [55], SST $k-\omega$ [56], RNG $k-\varepsilon$ [57, 58], and $\omega$-based Baseline Reynolds Stress model (BSL RSM) [59] are discussed in terms of the velocity and TKE fields inside the building and the airflow rate through the opening of the target building. Using statistical analysis, including the Monte Carlo Sampling technique and stochastic optimization methods, a discussion is presented to investigate the sensitivity of the CFD computations to the standard $k-\varepsilon$ closure coefficients. Development of a systematic approach to calibrate the CFD model 
based on the available measurement provides an opportunity to conduct a more accurate, more reliable, faster and easier CFD analysis using the RANS models compared with the LES models.

\section{Methodology}

\subsection{Mathematical modeling}

The 3D steady Reynolds averaged Navier-Stokes (RANS) equations is used to simulate the crossventilation behavior. These equations can be derived by substituting the mean and fluctuating components of the airflow variables into the Navier-Stokes equations [60]:

$$
\begin{aligned}
& \frac{\partial \rho}{\partial t}+\frac{\partial\left(\rho U_{j}\right)}{\partial x_{j}}=0 \\
& \frac{\partial\left(\rho U_{i}\right)}{\partial t}+\frac{\partial}{\partial x_{j}}\left(\rho U_{i} U_{j}\right)=-\frac{\partial P}{\partial x_{i}}+\frac{\partial}{\partial x_{j}}\left(\tau_{i j}-\rho \overline{u_{\imath} u_{J}}\right)+S_{M_{i}}
\end{aligned}
$$

where $U_{i}$ is the average velocity, $u_{i}$ is the fluctuating velocity, $\tau_{i j}$ is the viscous stress tensor (including both normal and shear components of the stress), $\rho \overline{u_{l} u_{J}}$ is the Reynolds (turbulent) stress and $S_{M_{i}}$ is the momentum source.

In this study two classes of turbulence models are used, including eddy viscosity and Reynolds stress models. In the eddy viscosity models, the Reynolds stresses can be related to the mean velocity gradients and eddy (turbulent) viscosity by the gradient diffusion hypothesis, in a manner analogous to the relationship between the stress and strain tensors in laminar Newtonian flow:

$$
-\rho \overline{u_{\imath} u_{\jmath}}=\mu_{t}\left(\frac{\partial U_{i}}{\partial x_{j}}+\frac{\partial U_{j}}{\partial x_{i}}\right)-\frac{2}{3} \delta_{i j}\left(\rho k+\mu_{t} \frac{\partial U_{k}}{\partial x_{k}}\right)
$$

where $\mu_{t}$ is the eddy viscosity or turbulent viscosity and $k$ is the turbulent kinetic energy. In the two-equation models, the turbulent velocity scale is computed from the TKE. Moreover, the turbulent length scale is estimated from TKE and its dissipation rate. The TKE and its dissipation rate are provided from the solution of their transport equations. In eddy viscosity models the effective viscosity is defined as:

$\mu_{\text {eff }}=\mu+\mu_{t}$

where $\mu$ is the molecular viscosity and $\mu_{\text {eff }}$ is the effective viscosity. The turbulence viscosity can be calculated via the following relationship:

$\mu_{t}=C_{\mu} \rho \frac{k^{2}}{\varepsilon}$

For the standard $k-\varepsilon$ model, values of $k$ and $\varepsilon$ can be directly extracted from their differential transport equations:

$\frac{D(\rho k)}{D t}=\frac{\partial}{\partial x_{j}}\left[\left(\mu+\frac{\mu_{t}}{\sigma_{k}}\right) \frac{\partial k}{\partial x_{j}}\right]+P_{k}-\rho \varepsilon$ 
$\frac{D(\rho \varepsilon)}{D t}=\frac{\partial}{\partial x_{j}}\left[\left(\mu+\frac{\mu_{t}}{\sigma_{\varepsilon}}\right) \frac{\partial \varepsilon}{\partial x_{j}}\right]+\frac{\varepsilon}{k}\left(C_{\varepsilon 1} P_{k}-C_{\varepsilon 2} \rho \varepsilon\right)$

where $C_{\mu}, \sigma_{k}, \sigma_{\varepsilon}, C_{\varepsilon 1}$ and,$C_{\varepsilon 2}$ are the model constants while $P_{k}$ is the viscous stress production term. Values of above parameters, which are the default values for most of the commercial CFD software, are as follows [61]:

$$
C_{\mu}=0.09, C_{\varepsilon 1}=1.44, C_{\varepsilon 2}=1.92, \sigma_{k}=1, \sigma_{\varepsilon}=1.3
$$

Furthermore, Reynolds stress models are calculated based on the transport equations for all components of the Reynolds stress tensor and the dissipation rate [60]. These models do not use the eddy viscosity hypothesis, but solve an equation for the transport of Reynolds stresses in the fluid. The exact production term and the inherent modeling of the stress anisotropies make Reynolds Stress models to be more theoretically suitable for the complex flows [44, 62]. A separate transport equation must be solved for each of the six Reynolds stress components of $\rho \overline{u_{\imath} u_{\jmath}}$. The differential equation of Reynolds stress transport thus can be represented as below [60]:

$\frac{\partial \rho \overline{u_{l} u_{J}}}{\partial t}+\frac{\partial}{\partial x_{k}}\left(U_{k} \rho \overline{u_{l} u_{\jmath}}\right)-\frac{\partial}{\partial x_{K}}\left(\left(\delta_{k l} \mu+\rho C_{S} \frac{k}{\varepsilon} \overline{u_{k} u_{l}}\right) \frac{\partial \overline{u_{l} u_{J}}}{\partial x_{l}}\right)=P_{i j}-\frac{2}{3} \delta_{i j} \rho \varepsilon+\varphi_{i j}+P_{i j, b}$

where $P_{i j}$ and $P_{i j, b}$ are the shear and buoyancy turbulence production terms of the Reynolds stresses, respectively. $\varphi_{i j}$ is the pressure-strain tensor, and $C_{s}=0.22$ is the equation constant. In this study, ANSYS CFX BSL Reynolds Stress model is used for the initial case study, which is based on the $\omega$ equation and automatic wall treatment. The constants of the RANS turbulence models are the default values provided in ANSYS CFX formulations, which are later modified with optimization technique to enhance the simulation results.

\subsection{The closure coefficients for the standard $k-\varepsilon$ model}

In this section, a very short description about the closure coefficients of the standard $k-\varepsilon$ model and their relations are presented. The goal is to show the uncertainty of the closure coefficients and the reported range of them in literature. Detailed description of the underlying physics for calculating the closure coefficients can be found in [44, 63]. As described in [44], the default values of the closure coefficients in the standard $k-\varepsilon$ model are obtained from a compromise to enable the model to perform for a variety of the airflow problems. The classical way to find the associated values is derived based on a series of fundamental flows. The value of $C_{\varepsilon 2}$ is calculated based on a homogeneous, isotropic, decaying turbulence assumption of an ideal flow in which $k$ and $\varepsilon$ equations become [45]:

$\frac{d k}{d t}=-\varepsilon$
$\frac{d \varepsilon}{d t}=-C_{\varepsilon 2} \frac{\varepsilon^{2}}{k}$

An analytical solution for these equations is $k(t)=k_{0}\left(\frac{t}{t_{0}}\right)^{-n}$ in which $t_{0}=n k_{0} / \varepsilon_{0}$ is the reference time and $n$ can be defined as $n=\frac{1}{C_{\varepsilon 2}-1}$; this led to $C_{\varepsilon 2}=\frac{n+1}{n}$. In literature, different values 
for $n$ and thus $C_{\varepsilon 2}$ are reported. Standard value for $C_{\varepsilon 2}$ is 1.92 , which is a default value for the commercial software such as ANSYS CFX, ANSYS FLUENT, STAR-CCM+, and PHOENIX. Default value of $C_{\varepsilon 2}$ for $R N G k-\varepsilon$ is 1.68 while Mohamed and Larue [50] suggested a value of $C_{\varepsilon 2}=$ 1.77 .

The value of $C_{\mu}$ calculated based on the balance between the production and dissipation in the simple turbulent shear flow, which can be expressed as below:

$P_{k}=\mu_{t}\left(\frac{\partial U_{1}}{\partial x_{3}}\right)^{2}=C_{\mu} \frac{k^{2}}{\varepsilon}\left(\frac{\partial U_{1}}{\partial x_{3}}\right)^{2}=\varepsilon$

Using the eddy-viscosity approximation $\left(\overline{u_{1} u_{3}}=v_{t} \frac{\partial U_{1}}{\partial x_{3}}\right)$, the coefficient can be obtained as $C_{\mu}=$ $\left(\frac{\overline{u_{1} u_{3}}}{k}\right)^{2}$. Experimental and numerical analyses by Kim et al. [64] show that the variation of $C_{\mu}$ in areas far from the wall $\left(y^{+}>50\right)$ is between 0.06 to 0.095 , resulting in an average value of $C_{\mu}=0.09$. The value of this quantity for a temporal-mixing layer, except near the edge, was reported between 0.07 and $0.11[44]$.

The relationship between $C_{\varepsilon 1}$ and $C_{\varepsilon 2}$ can be also found from the homogeneous shear flow properties, which results in $\frac{P_{k}}{\varepsilon}=\frac{C_{\varepsilon 2}-1}{C_{\varepsilon 1}-1}$ [44]. Experiments done by Tavoulakis and Karnik [51] for different shear flows resulted in different values for $\frac{P_{k}}{\varepsilon}$, ranging from 1.33 to 1.75 . This ratio is $\frac{P_{k}}{\varepsilon}=$ 2.09 when default values of $C_{\varepsilon 1}$ and $C_{\varepsilon 2}$ are used, which is noticeably different from the reported experiment value [45]. Another constraint for the closure coefficients is based on the physics of the fully developed channel flow in log-law region that results in [44]:

$\sigma_{\varepsilon}=\frac{\kappa^{2}}{C_{\mu}^{1 / 2}\left(C_{\varepsilon 2}-C_{\varepsilon 1}\right)}$

where $\kappa$ is the Von Karman constant, varying from 0.33 to 0.45 [65]. The value of $\sigma_{k}=1$ is also proposed by Launder and Spalding [61]. According to [45, 52], there is no data or experiment available to define a suitable range for this constant. The value of this constant for the $R N G k-\varepsilon$ model is $\sigma_{k}=0.7179$ in ANSYS CFX formulation.

\subsection{Description of the cross-ventilation experiment}

Experimental data by Tominaga and Blocken [1] are used in this study to validate the microclimate CFD model. The experiment was conducted to analyze the cross-ventilation of an unsheltered and sheltered building in an isothermal boundary layer wind tunnel as shown in Fig. 1(a). Target building has a dimension of $W \times D \times H=0.2 m \times 0.2 m \times 0.16 m$ with two openings of the similar size $(0.092 \mathrm{~m} \times 0.036 \mathrm{~m})$ on opposite walls. The thickness of walls and ceiling is $3 \mathrm{~mm}$.

In the case of the sheltered building, eight similar cuboid buildings without opening are surrounding the target building at a distance equals to the building width $(W)$. Location of the measurement points in the central vertical section of the building is also displayed in Fig. 1(b). Flow 
data at these measurement points, including velocity and TKE, are used for the CFD validation and statistical optimization.

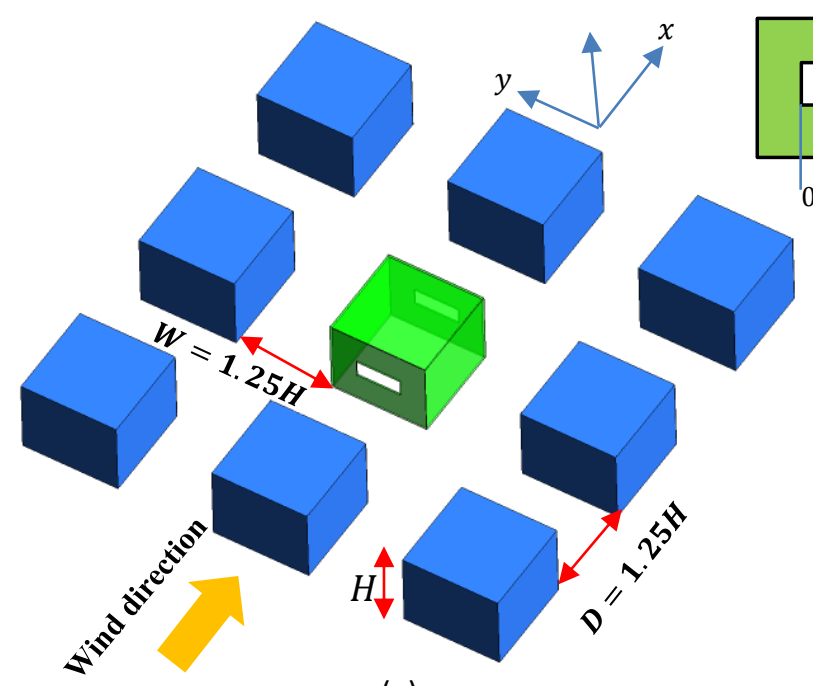

(a)

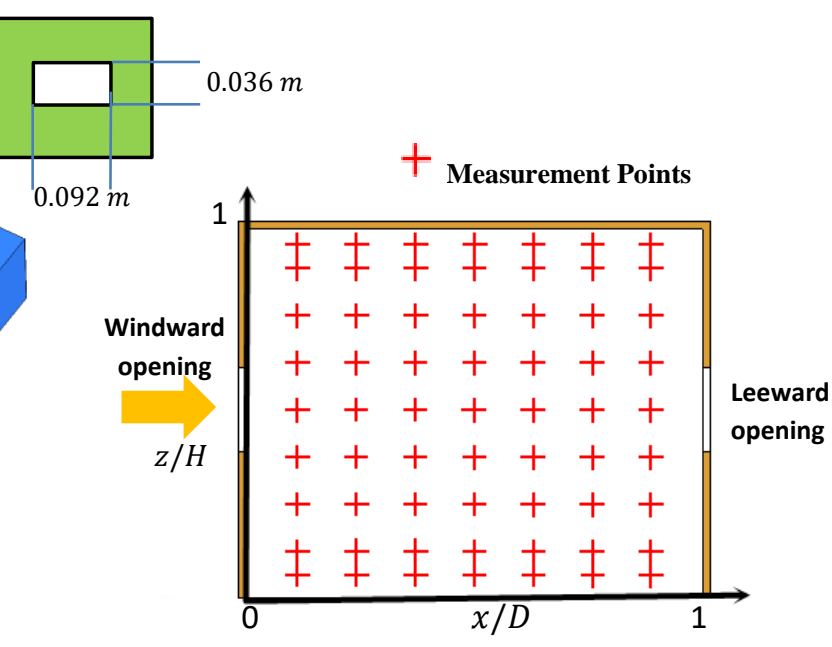

(b)

Figure 1 (a) Configuration of the sheltered building and (b) measurement points in the centeral vertical section.

\subsection{Outline of CFD domain and setup}

A rectangular computational domain, as shown in Fig. 2, was considered based on the recommendations by AIJ guidelines [66] and similar studies [8, 16]. The domain width, length, and height were $2.6 m \times 4.2 m \times 0.96 m$. ICEM CFD meshing package was used to create the structured hexahedral mesh using the blocking technique. Boundary conditions were implemented at inlet boundary directly from the experiment; including vertical profiles of the streamwise velocity and TKE (see Fig. 3). A power law profile was used for the inlet velocity, which represents the wind profile at the lower part of a neutral atmospheric boundary layer:

$\frac{U(z)}{U_{H}}=\left(\frac{Z}{H}\right)^{0.25}$

where $U(z)$ is the streamwise velocity at the height of $z$, and $U_{H}=4.3 \mathrm{~m} / \mathrm{s}$ is the velocity at the building height $H$. The measured vertical profile of the TKE was also approximated by an exponential formulation proposed in [1]:

$\frac{k(z)}{U_{H}^{2}}=0.033 \exp ^{-0.32(z / H)}$

The turbulent kinetic energy dissipation rate $\varepsilon(z)$ was approximated in accordance with the AIJ guidelines [66]:

$\varepsilon(z)=C_{\mu}^{\frac{1}{2}} k(z) \frac{U_{H}}{H} \alpha\left(\frac{Z}{H}\right)^{\alpha-1}$

where $C_{\mu}$ denotes the model constant equals to 0.09 and $\alpha=0.25$. 

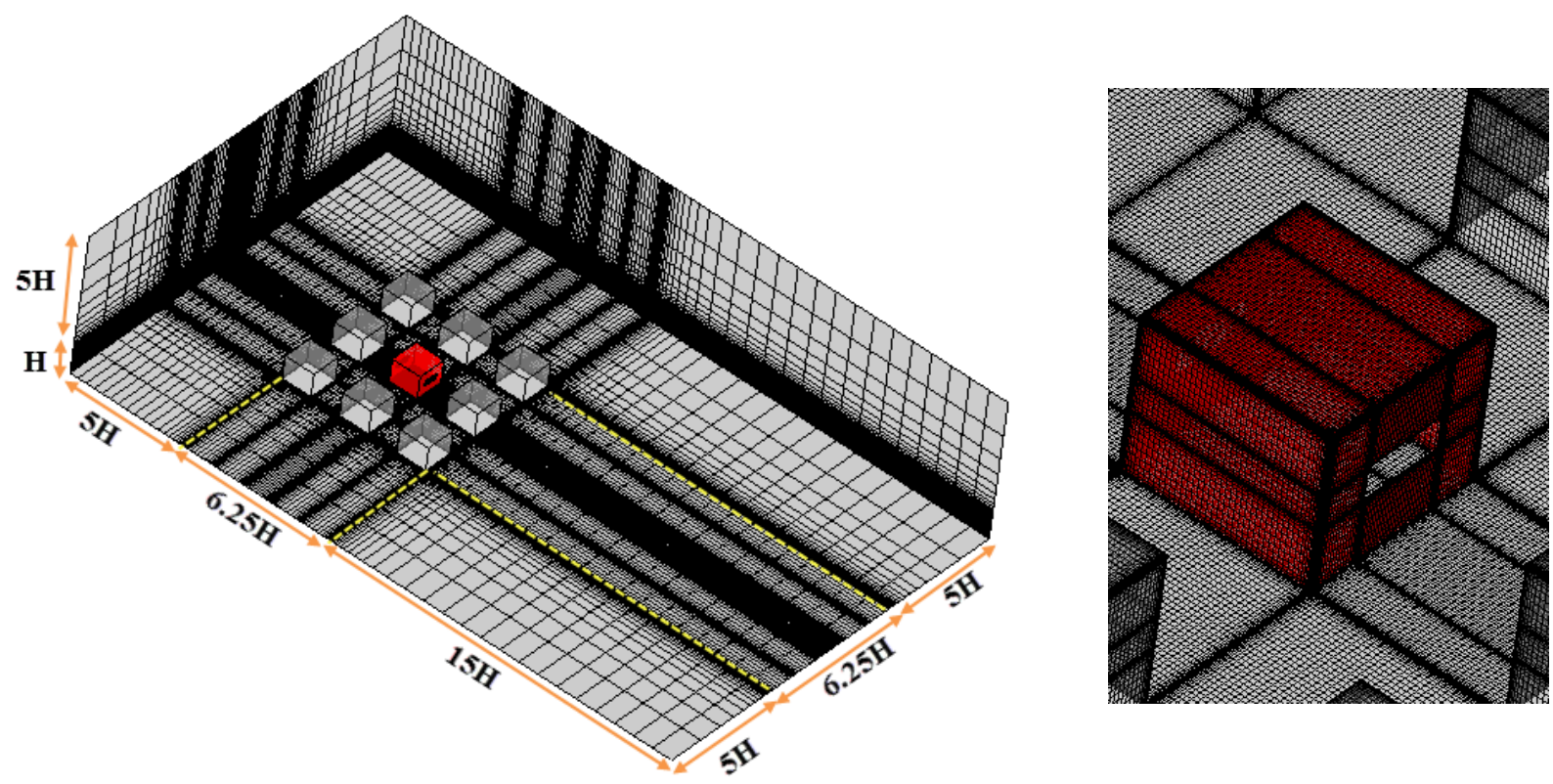

Figure 2 Computational domain and grid arrangement

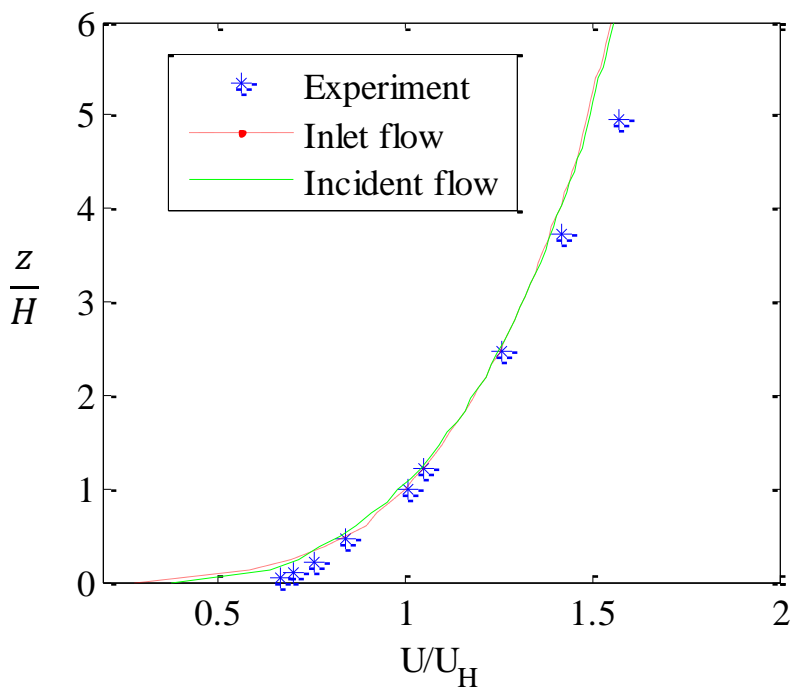

(a)

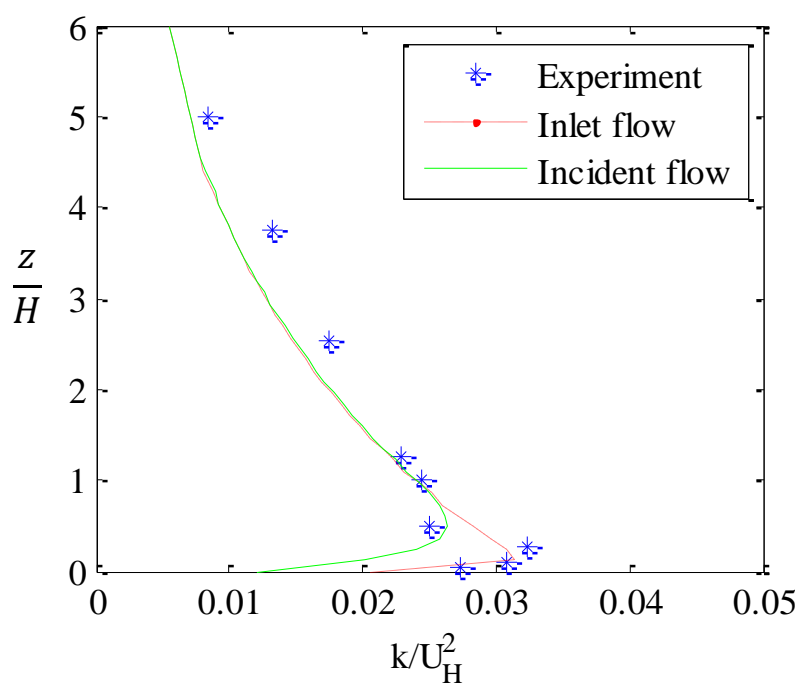

(b)

Figure 3 Vertical profile of (a) the time averaged streamwise velocity and (b) turbulent kinetic energy of the experiment, and inlet flow and incident flow from the empty-domain test case.

Due to the gradual change of the vertical wind velocity profile in the wind tunnel, an emptydomain CFD simulation test was first performed with the same grid distribution in the vertical direction equals to the main CFD model. Mean velocity and TKE vertical profiles of the inlet and incident flows are depicted in Fig.3 for the empty domain test case. There was no streamwise inhomogeneity observed in the vertical profile of the mean velocity, however, a streamwise gradient in the vertical profile of the TKE was found near the ground surface at ${ }^{Z} / H<0.5$. This streamwise inhomogeneity seems acceptable as the TKE at the reference height is equal to that of the inlet flow [8]. A no-slip smooth boundary condition was also considered for the ground and building surfaces. 
Symmetric wall boundary conditions were assigned to the lateral boundary surfaces while a free-slip wall boundary condition was assumed for the top boundary surface.

The RANS equations were solved using the commercial software ANSYS CFX, which uses an element-based finite volume discretization method. Two near-wall modeling approaches, including scalable and automatic wall functions, were used depending on the utilized turbulence model. Scalable wall function is based on the modification of the Launder and Spalding [55] to treat roughness of walls. In automatic wall function approach available in ANSYS CFX, wall treatment automatically switches from wall-functions to a low-Re near wall formulation as the mesh is refined. For building surfaced an average $Y^{+} \approx 1$ was applied while an expansion ratio of about 1.2 and a first layer size of $0.0005 \mathrm{~m}$ were considered for the boundary layer meshes. The CFD solver iterations have been continued until reaching residuals of less than $10^{-4}$ for momentum, $k$ and $\varepsilon$ equations.

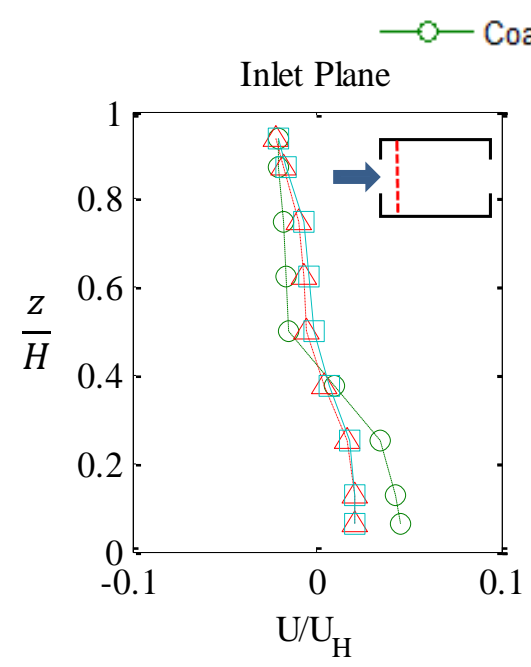

(a)

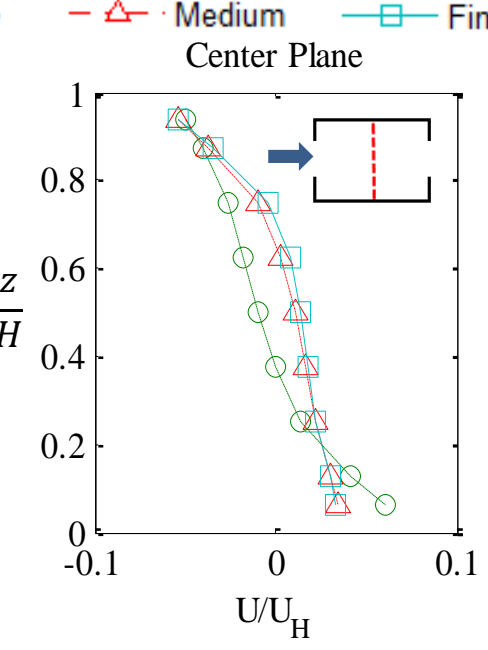

(b)

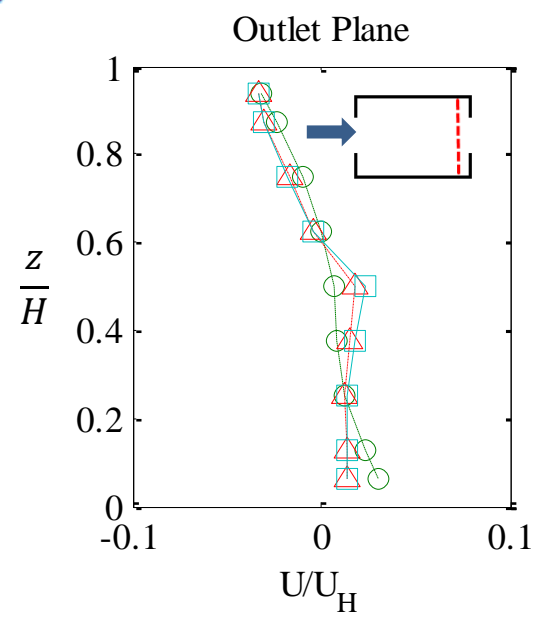

(c)

Figure 4 Vertical distribution of the mean streamwise velocity inside the sheltered building at: (a) $\frac{x}{D}=0.125,(b) \frac{x}{D}=0.5,(c) \frac{x}{D}=0.875$.

A mesh sensitivity study was conducted for the case of the sheltered building to find a meshindependent grid. The number of cells for coarse, medium and fine meshes were 2,332,870 and $4,040,656$ and $6,998,621$, respectively. The vertical distribution of the mean streamwise velocity for different mesh settings at three different positions, i.e. inlet plane $(x / D=0.125)$, center plane $(x / D=$ $0.5)$, and outlet plane $(x / D=0.875)$, is shown in Fig. 4. The standard $k-\varepsilon$ model was used for the mesh sensitivity analysis. The sensitivity of the velocity profile to the cell size was found to be very low after refinement of the medium mesh and hence it was used for the rest of the calculations.

\subsection{CFD validation of the cross-ventilation for an isolated building model}

In order to show the reliability of the CFD simulation, a separate simulation was initially performed for the case of unsheltered building. The numerical results were compared with an experimental study by Tominaga and Blocken [1] and a numerical analysis presented by Hoof et al. [25] where ANSYS FLUENT was utilized as the CFD solver. Similar CFD setting as [25] was considered in this study though ANSYS CFX was used as the solver. Vertical distribution of the mean 
streamwise velocity $\left(U / U_{H}\right)$ at three different positions $(x / D=0.125, x / D=0.5, x / D=0.875)$ is illustrated in Fig. 5 and it can be seen that the numerical results of current study and those of van Hooff et al. [25], which are both based on the $R N G k-\varepsilon$ model, show a very good agreement with the experimental data. It was also observed that the value of the streamwise velocity gradient $\partial \overline{\mathrm{U}} / \partial \mathrm{z}$ formed at the inlet opening around the incoming jet decreases as the flow approaching to the leeward opening where $\partial \overline{\mathrm{U}} / \partial \mathrm{z}$ reaches to its minimum value.

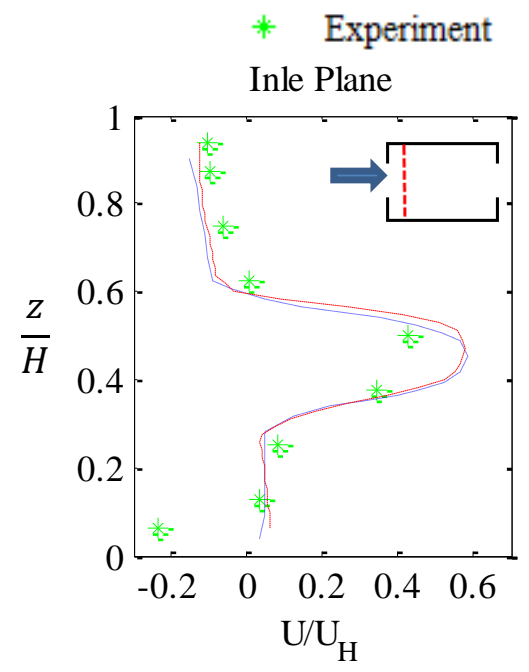

(a)

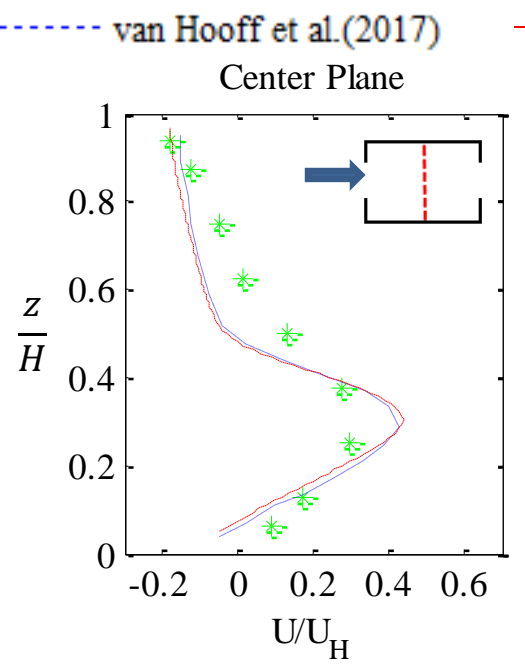

(b)

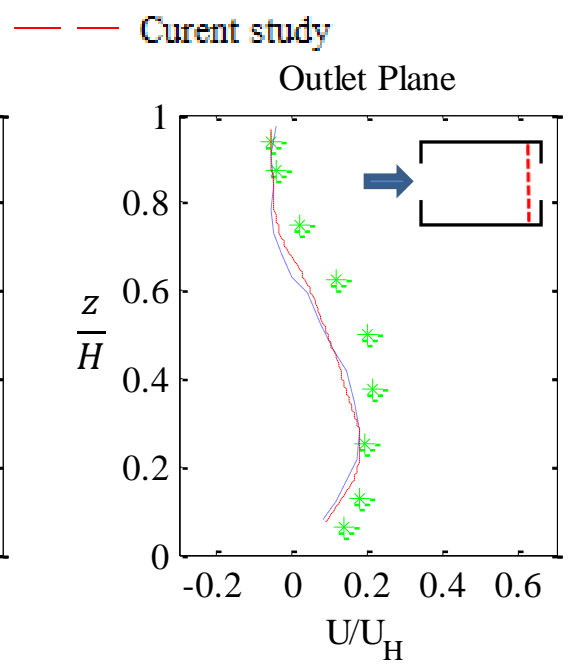

(c)

Figure 5 Vertical distribution of the mean streamwise velocity inside the unsheltered building at: (a) $\frac{x}{D}=0.125,(b) \frac{x}{D}=0.5$, , (c) $\frac{x}{D}=0.875$.

\subsection{CFD validation of the cross-ventilation in the sheltered building}

The vertical distribution of the streamwise velocity calculated by different RANS turbulence models at three different streamwise positions for the sheltered building scenario is shown in Fig. 6 and results are compared with those of the experiment. In the case of the sheltered building scenario, the incoming jet velocity shows a noticeable decrease in comparison with the unsheltered case (see Fig. 5), which can be explained as the blocking effect of the surrounding buildings. For the sheltered building scenario, the level of agreement between the experimental and CFD data for all the RANS turbulence models are very poor in contrast to the unsheltered building scenario. At the windward plane $\left(\frac{x}{D}=0.125\right)$, the standard $k-\varepsilon, S S T$ and $R N G$ models fail to capture the velocity profile of the incoming jet. The BSL RSM model accurately predicts the velocity profile at the lower part of the building, but it over-predicts the velocity in the upper part of the building similar to other turbulence models. The same mismatch between the experimental and CFD data can be observed in the center plane $\left(\frac{x}{D}=0.5\right)$ where the standard $k-\varepsilon, S S T$ and $R N G$ models predict a constant velocity profile. In the lower part of the building, the $B S L R S M$ model shows more accurate results. In the leeward plane $\left(\frac{x}{D}=0.875\right)$, the standard $k-\varepsilon$ and $B S L R S M$ models predict similar velocity profiles and both overestimate the streamwise velocity in the upper part of the building. However, in the leeward plane, the $S S T$ and $R N G$ models resolve a very different pattern for the streamwise velocity where flow is simulated to inversely enter the building through the leeward opening. 


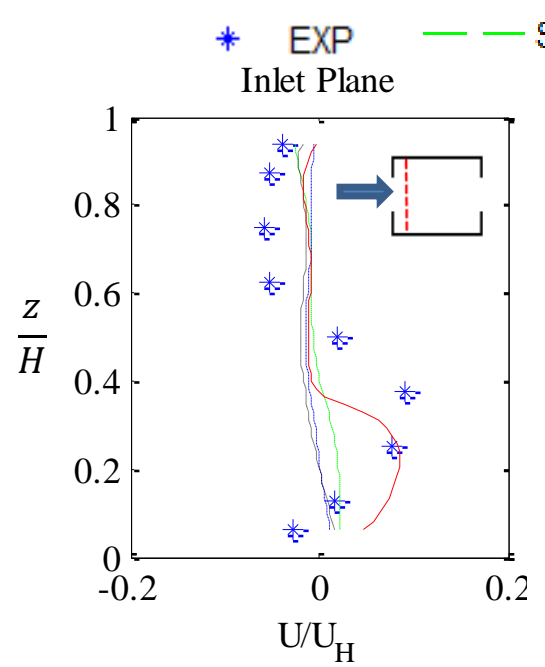

(a)

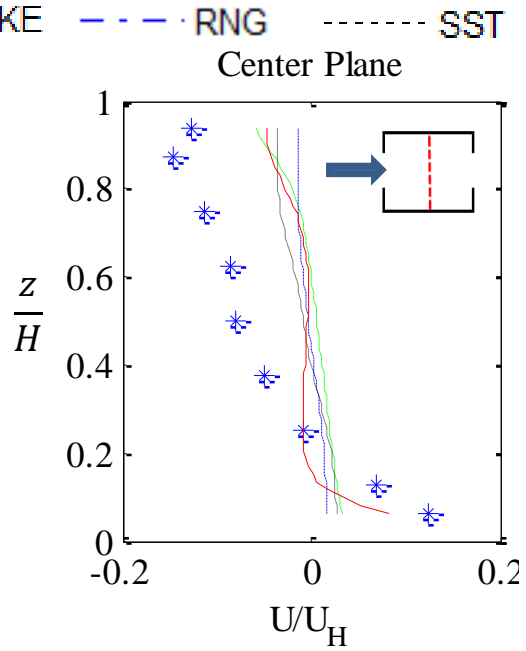

(b)

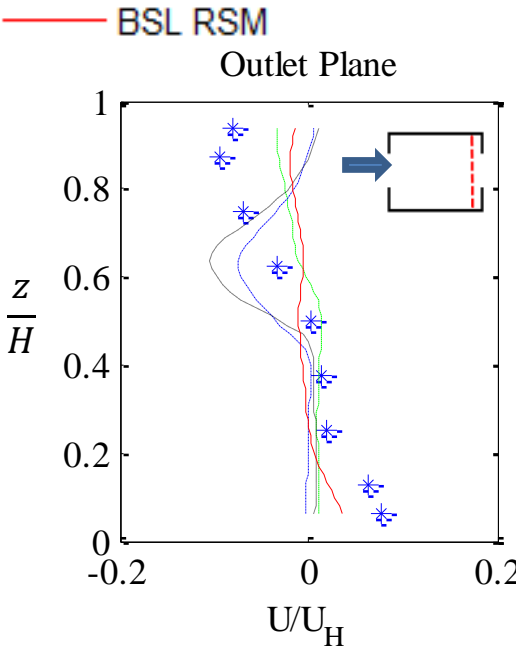

(c)

Figure 6 Comparison of vertical distribution of the mean streamwise velocity obtained by experiment, the standard $\boldsymbol{k}-\varepsilon(S K E), \boldsymbol{R N G}, \mathbf{S S T}$, and BSL RSM models inside the sheltered building at: (a) $\frac{x}{D}=\mathbf{0 . 1 2 5}$, (b)

$$
\frac{x}{D}=0.5,(c) \frac{x}{D}=0.875 \text {. }
$$

To have a general picture of the flow pattern inside the sheltered building, streamlines are plotted as demonstrated in Fig. 7 for four different turbulence models, including the standard $k-\varepsilon, S S T, R N G k-$ $\varepsilon$, and $B S L R S M$. Focusing on the streamlines from the experiment, it can be seen that the crossing jet through the windward opening is much weaker than that of the unsheltered building shown in Tominaga and Blocken [1] . It is mainly due to the flow distraction effect of the upstream building, which forces the airflow moving downward in the vortex generated in its wake region. Inside the building section, a large counter-clockwise recirculation is formed while its center is near the leeward opening. For the eddyviscosity based models, i.e. the standard $k-\varepsilon, S S T, R N G k-\varepsilon$, a very large single counter-clockwise recirculating flow is predicted although its center is miscalculated far from the leeward opening, and placed in the center of the building. In contrast to the eddy-viscosity based models, the BSL RSM predicts a more similar flow pattern to the experiment, and it can be seen that the center of the recirculation is close to the leeward opening. However, the direction of the streamlines between the windward opening and the center of the building is not similar to that of the experiment where the flow is more vertically inclined. The above mentioned discrepancies between different turbulence models are mainly originated from the accuracy of these models in the prediction of the roof separation/reattachment and the wake formed behind the upstream building, and also due to their inherent poor accuracy in prediction of the TKE distribution inside the street canyon. In fact, low accuracy of the steady RANS models in understanding of the momentum diffusion in the wake region between the upstream and downstream adjacent buildings results in inaccurate calculation of cross-ventilation through the target building. 


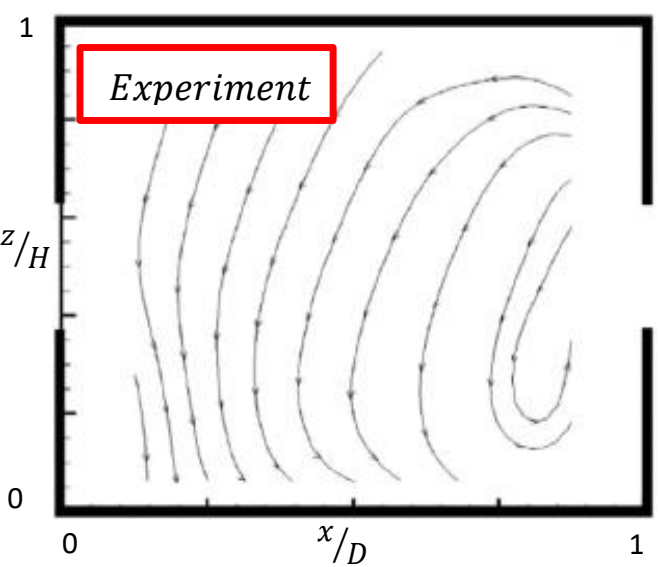

(a)

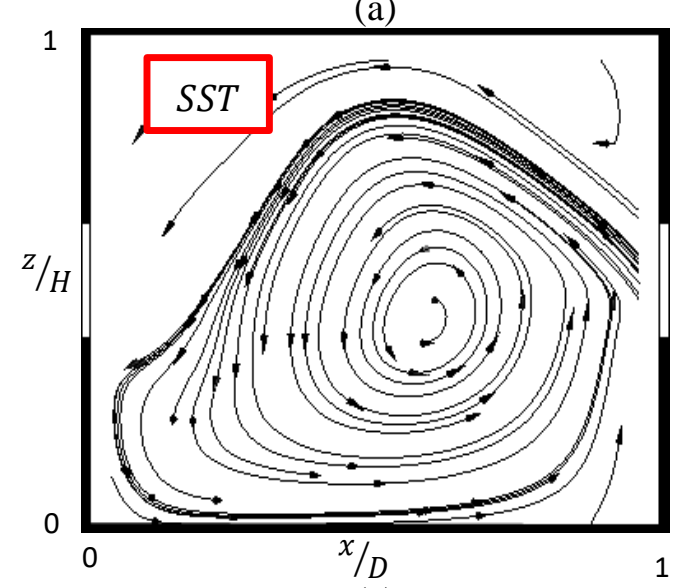

(c)

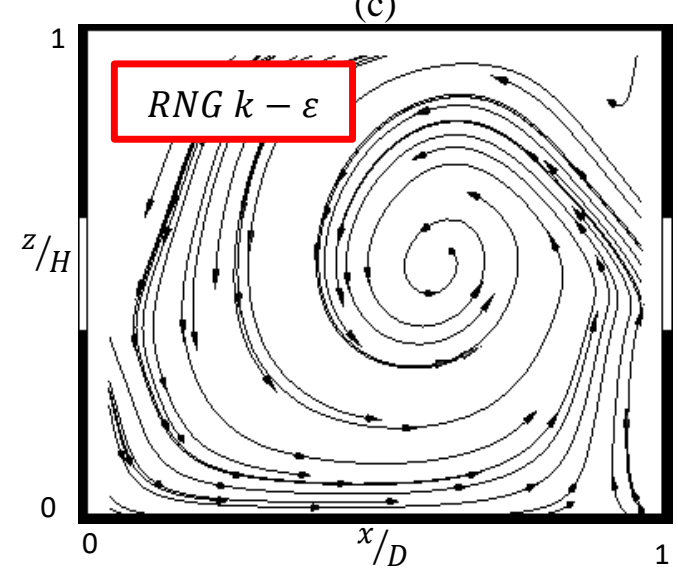

(e)

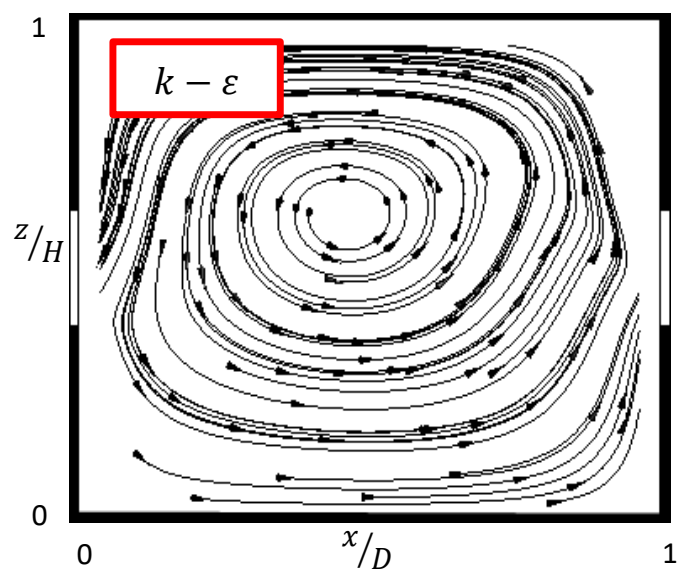

(b)

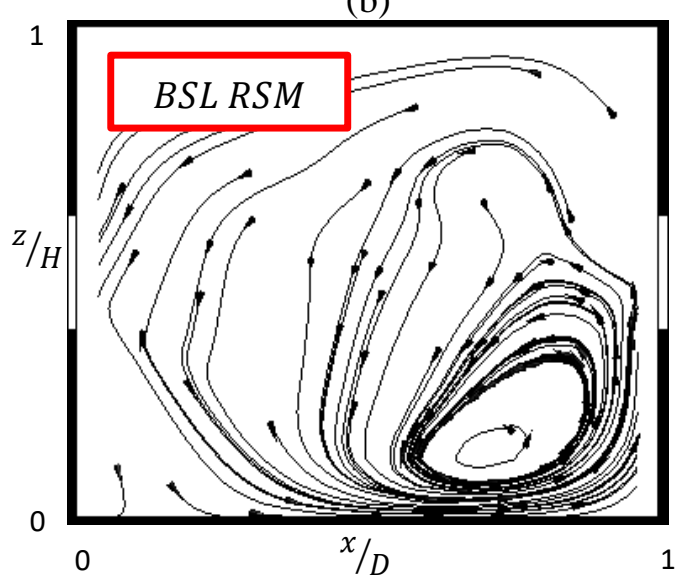

(d)

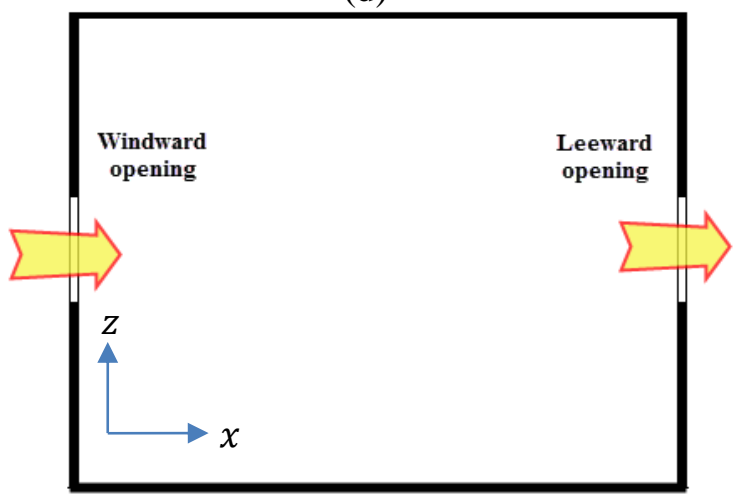

(f)

Figure 7 Streamlines in the vertical center section of the sheltered building: (a) experimental data by Tominaga and Blocken [1], (b) standard $\boldsymbol{k}-\boldsymbol{\varepsilon}$, (c) SST, (d) BSL RSM, (e) RNG $\boldsymbol{k}-\boldsymbol{\varepsilon}$.

Spatial average of the measured TKE distribution inside the sheltered building scenario was reported to be $43 \%$ lower than that was measured for the unsheltered building scenario [1]. Results of the validation study for the unsheltered building showed that the CFD prediction of the TKE inside the building was lower than the experimental data reported in [1]. This inaccuracy was even worse in the case of the sheltered building scenario. In Table 1 , average of the normalized TKE $\left(k / U_{H}^{2}\right)$ at three streamwise positions inside the building model are shown for different turbulence models, and 
compared with those of the experiment. It can be evidently seen that all turbulence models underpredict the TKE at least one order of magnitude lower than the experimental data.

Table 1 Spanwise averaged turbulent kinetic energy at three different streamwise positions inside the sheltered
building
\begin{tabular}{ccccccc}
$k / U_{H}^{2} \times 10^{4}$ & Experiment & $k-\varepsilon$ & $R N G$ & $S S T$ & BSL RSM \\
\hline$\frac{x}{D}=0.125$ & 211 & 2.21 & 1.45 & 2.1 & 8.19 \\
$\frac{x}{D}=0.5$ & 67 & 2.24 & 1.23 & 3.65 & 1.02 \\
$\frac{x}{D}=0.875$ & 69 & 10.4 & 15.2 & 17.2 & 1.05
\end{tabular}

Value of the calculated non-dimensional airflow rate $\frac{Q}{U_{H} A_{\text {inlet }}}$ for the sheltered building condition is shown in Table 2 for the standard $k-\varepsilon, S S T, R N G k-\varepsilon$, and BSL RSM. These values are compared with that was reported in [1]. The accuracy of the CFD model in prediction of the airflow rate of the cross-ventilation varies according to the selected turbulence model. For the given geometry and boundary conditions, the eddy viscosity-models fail to predict the airflow rate value and its direction through the openings of the sheltered building scenario. The experimental airflow rate is reported in [1] to be 0.07 while its value for the case of the standard $k-\varepsilon$ is -0.0004 . The negative sign for the non-dimensional airflow rate means that the predicted airflow enters the building through the leeward opening instead of the windward opening. Estimated airflow rates for $R N G k-\varepsilon$ and $S S T$ were -0.0052 and -0.0012 , which both are incorrect in terms of magnitude and direction. The $B S L R S M$ model provided a more accurate velocity distribution and airflow rate. However, the $B S L R S M$ model significantly under-predicted the airflow rate crossing the openings with the value equal to 0.0162 . Here, it is important to be noted that the direction of the measured airflow rate during the experiment cannot be determined explicitly as the tracer gas technique was used for measuring the volume flow rate. The accuracy of the airflow rate measurement in the experiment was reported to be $\pm 7 \%[25]$.

Table 2 Non-dimensional airflow rate $\frac{Q}{U_{H} A_{\text {inlet }}}$ for the sheltered building scenario

\begin{tabular}{cccccc}
$\begin{array}{c}\text { Non- } \\
\text { dimensional } \\
\text { airflow rate }\end{array}$ & $\begin{array}{c}\text { Experimental } \\
\text { data }\end{array}$ & $\begin{array}{c}\text { Standard } \\
k-\varepsilon\end{array}$ & RNG $k-\varepsilon$ & SST & BSL RSM \\
\hline$\frac{Q}{U_{H} A_{\text {inlet }}}$ & 0.07 & -0.0004 & -0.0052 & -0.0012 & 0.0162
\end{tabular}

To understand the reason of such inaccuracy in predicting crossing airflow rate for the RANS models, the distribution of pressure coefficient $\left(C_{p}=\frac{P-P_{r e f}}{0.5 \rho U_{H}^{2}}\right)$ along a horizontal line starting from the mid height of the leeward surface of the upstream building and ending at the windward surface of the downstream building is plotted as shown in Fig.8. The estimated pressure differences by the standard $k-\varepsilon, R N G$, and $S S T$ turbulence models are very low at the windward and leeward openings of the target building; hence, an incorrect airflow direction and airflow rate were predicted at these locations. Results of the BSL RSM model showed a better performance in the calculation of the airflow direction. In the $B S L R S M$ model, a noticeable pressure difference is estimated for the 
windward and leeward openings, which has forced the air to move through the windward opening and moves out of the leeward opening. The variation of the internal pressure inside the building $(0 \leq$ $x / D \leq 1)$ is almost constant although its average value varies for different RANS models. It is noteworthy to mention that the stability of the numerical results using steady Reynolds Stress models was a challenging issue in the case of the sheltered building. Utilization of the Reynolds Stress models requires careful considerations of the convergence issue. Furthermore, it was noticed that the fluctuating behavior of the flow parameters became more challenging when a finer mesh was used for the BSL RSM model.

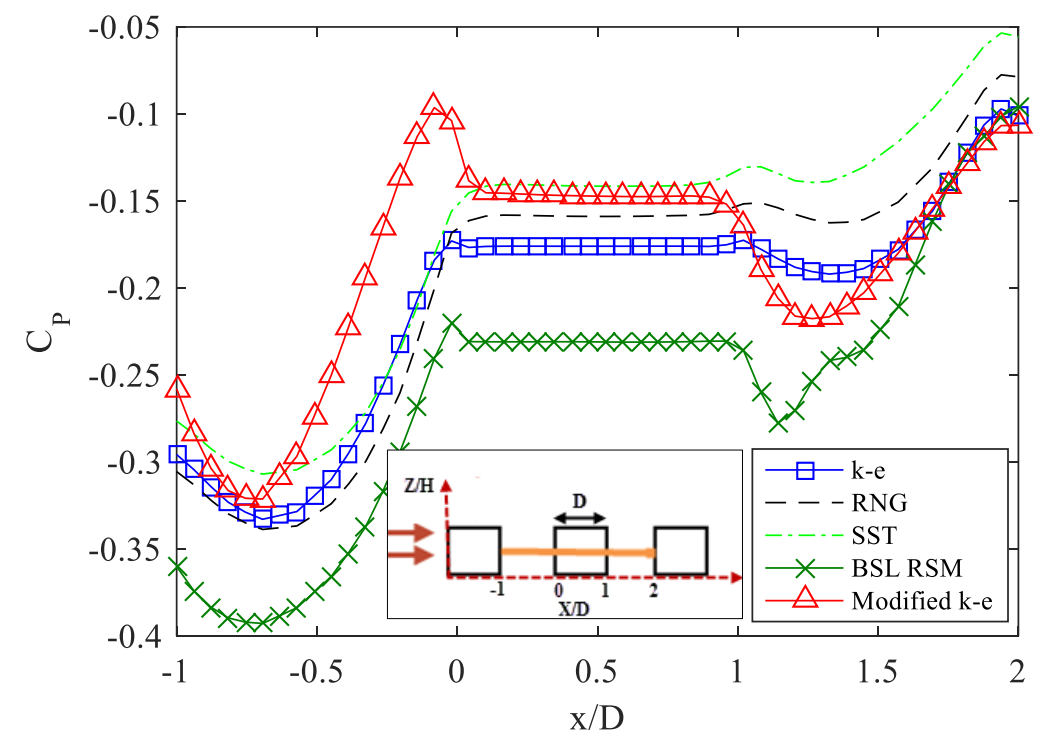

Figure 8 Pressure coefficient $\left(C_{P}=\frac{P-P_{\text {ref }}}{0.5 \rho U_{H}^{2}}\right)$ along a horizontal line starting from the upstream building leeward face to the downstream building windward face through the openings.

\section{Improving of the turbulence modeling accuracy using stochastic optimization}

As it was shown in the previous section, accuracy of the steady RANS turbulence models for cross-ventilation modeling of the sheltered building scenarios is generally poor. Because of the limitations of the unsteady RANS and LES models, it is desirable to propose a method to improve the accuracy of the RANS models for CFD modeling of the sheltered cross-ventilation scenarios. To this end, a stochastic optimization of the closure coefficients is presented in this section to improve the accuracy of the standard $k-\varepsilon$ model.

The optimization method used in this study is based on the integration of the Monte Carlo Sampling (MCS) technique and the Nonlinear Programing with Non-Monotone and Distributed Line Search (NLPQLP) optimization method [67]. A schematic of the stochastic optimization methodology for the closure coefficients are depicted in Fig. 9. In the optimization process, the probability density function (PDF) of the closure coefficients are given as uncertainty variables into the program, then a series of CFD simulations performed automatically in order to characterize the statistical characteristics of the output variables, which in this case are validation metrics. 
The brief description of the formulation of stochastic optimization can be mathematically stated as finding a set of design variables $\mathrm{X}$ that [68]:

Minimize: $\quad f\left(\mu_{y}(X), \sigma_{y}(X)\right)$

Subject to: $\quad g_{i}\left(\mu_{y}(X), \sigma_{y}(X)\right) \leq 0$

$$
X_{L} \leq X \leq X_{U}
$$

where $X_{L}$ and $X_{U}$ are the lower and upper limits for input parameter $X$. In this formulation, the output constraint $g_{i}$ is expressed in terms of mean value and standard deviation. The objective function considered in this study was defined for four terms, including the mean value and standard deviation of the validation metrics. A weighted sum approach was used to define the objective function, which includes a term for mean value variation relative to the target and a term to minimize the standard deviation of the output responses [68]:

$$
F=\sum_{i=1}^{l}\left[\frac{w_{1_{i}}}{s_{1_{i}}}\left(\mu_{y_{i}}-M_{i}\right)^{2}+\frac{w_{2_{i}}}{s_{2_{i}}} \sigma_{y_{i}}^{2}\right]
$$

where $w_{1_{i}}$ and $w_{2_{i}}$ are the weighting factors, and $s_{1_{i}}$ and $s_{2_{i}}$ are the scale factors related to each term. $M_{i}$ stands for the target of the output response $i$ and $l$ is the total number of output responses. The statistical variability of output responses (i.e. $\mu_{y_{i}}$ and $\sigma_{y_{i}}$ ), which are required by the stochastic optimization formulation, can be estimated using Monte Carlo simulation (MCS) technique. The number of random CFD simulations generated in the Monte Carlo simulation are 50, compromising between the statistical analysis accuracy and the computational cost. A total of 250 CFD simulations were executed during the optimization process. Simulations were conducted using an 8-core AMD® CPU processor, which took about 4 days.

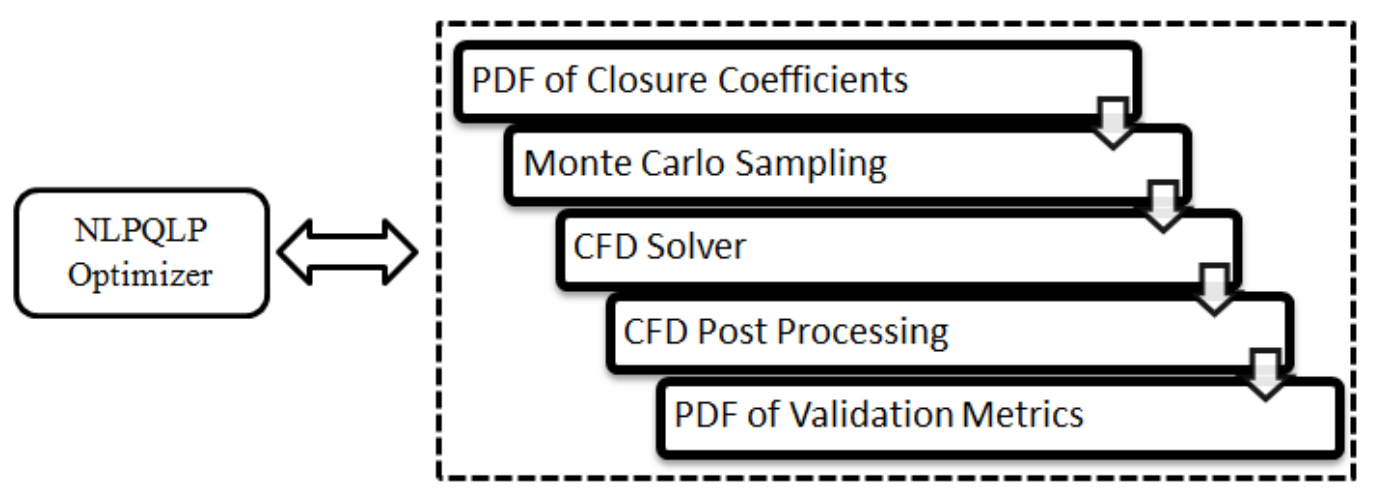

Figure 9 Schematic of the stochastic optimization of the closure coefficients of the standard $\boldsymbol{k}-\boldsymbol{\varepsilon}$.

In order to quantify the agreement between the experimental and CFD results, the below validation metrics are applied to the simulated scenarios [35]. These metrics are defined for both $U$ and $k$ variables at 63 measurement points shown in Fig. 1(b). These validation metrics, include the hit rate $q$, the fraction of the predictions within a factor of 2 of the observations (FAC2), the fractional bias $(F B)$, and the normalized mean square error $(N M S E)$ :

$q=\frac{1}{N} \sum_{i=1}^{N} n_{i} \quad$ if $\left|\frac{P_{i}-Q_{i}}{P_{i}}\right| \leq D_{q} \quad$ or $\left|P_{i}-Q_{i}\right| \leq W_{q} \quad n_{i}=1$ else $n_{i}=0$ 
$F B=\frac{[Q]-[P]}{0.5([Q]+[P])}$

$N M S E=\frac{\left[\left(Q_{i}-P_{i}\right)^{2}\right]}{[Q][P]}$

$F A C 2=\frac{1}{N} \sum_{i=1}^{N} n_{i} \quad n_{i}=1 \quad$ if $\quad 0.5 \leq \frac{P_{i}}{Q_{i}} \leq 2$ else $n_{i}=0$

where $O_{i}$ and $P_{i}$ are the measured and computed values of a given variable for sample $i$, respectively. $N=63$ is the number of data points used in the optimization process. The ideal value of the validation metrics for a complete agreement between the experimental and numerical results is 1 for $q$ and $F A C 2$, and 0 for $F B$ and NMSE.

\section{Result and discussion}

A parametric sensitivity study for cross-ventilation of the sheltered building is firstly conducted to obtain the effect of the CFD model outputs to the closure coefficients variation. Parametric sensitivity study is thus performed by varying the closure coefficients, i.e. $C_{\varepsilon 1}, C_{\varepsilon 2}, C_{\mu}$ and $\sigma_{k}$, in a specified range as shown in Table 3. The range of these coefficients is chosen with regards to the previous studies in literature and the result of the parametric study. Value of $\sigma_{\varepsilon}$ is calculated using Eq.12. In Table 3, the default value of the closure coefficients that are embedded in commercial CFD software such as ANSYS CFX, ANSYS FLUENT, and STAR-CCM+, and PHOENIX are also shown. For the parametric sensitivity analysis, 20 uniformly distributed samples were considered for each parameter in the specified range in Table 3.

Table 3 Default value and range of the closure coefficients for the parametric sensitivity study

\begin{tabular}{ccccc} 
& $C_{\varepsilon 1}$ & $C_{\varepsilon 2}$ & $\sigma_{k}$ & $C_{\mu}$ \\
\hline Standard value & 1.44 & 1.92 & & 0.09 \\
Ranges & $1.0-1.5$ & $1.5-3.2$ & $0.8-1.4$ & $0.05-0.15$
\end{tabular}




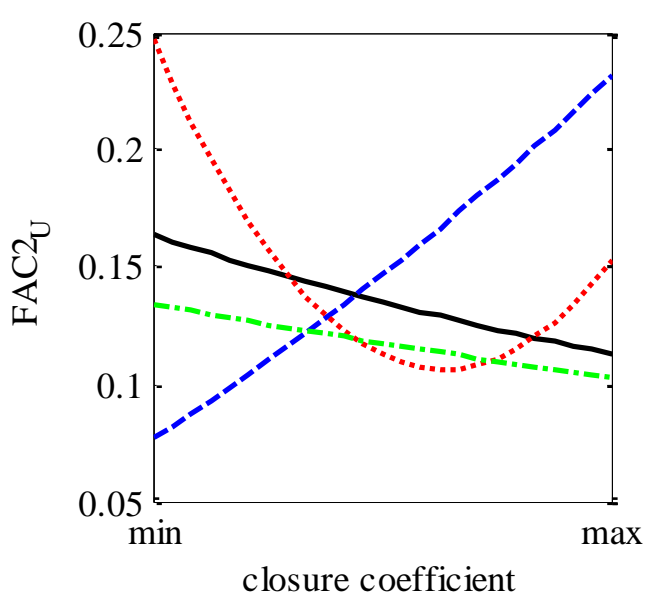

(a)

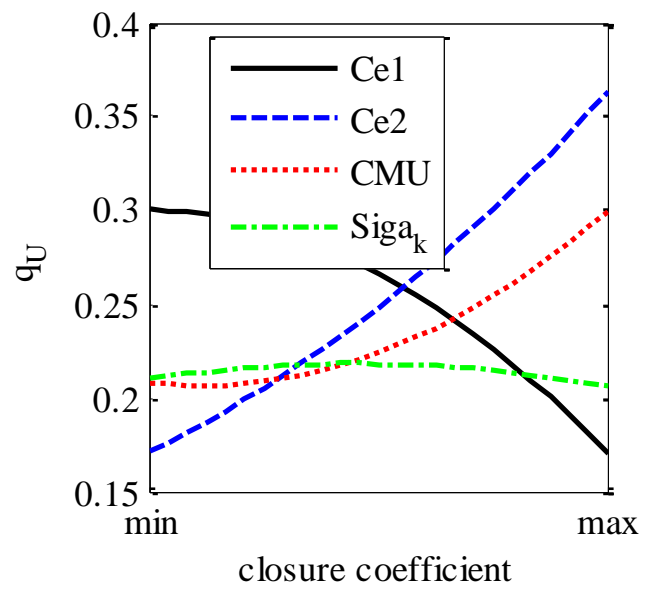

(c)

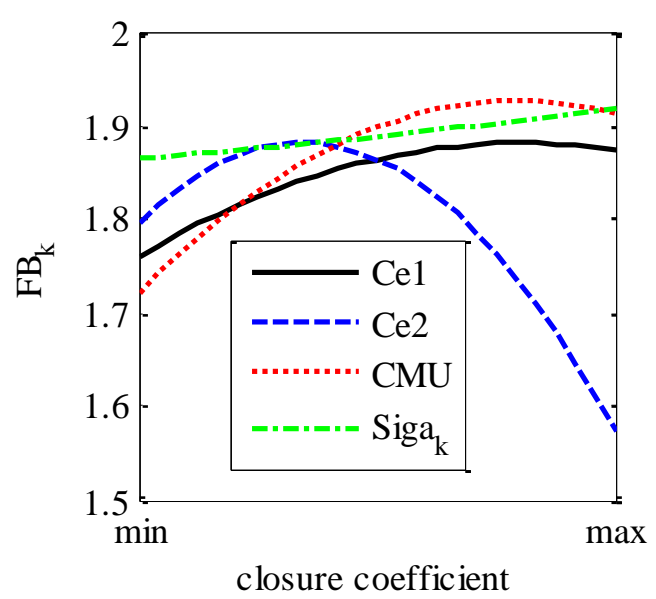

(b)

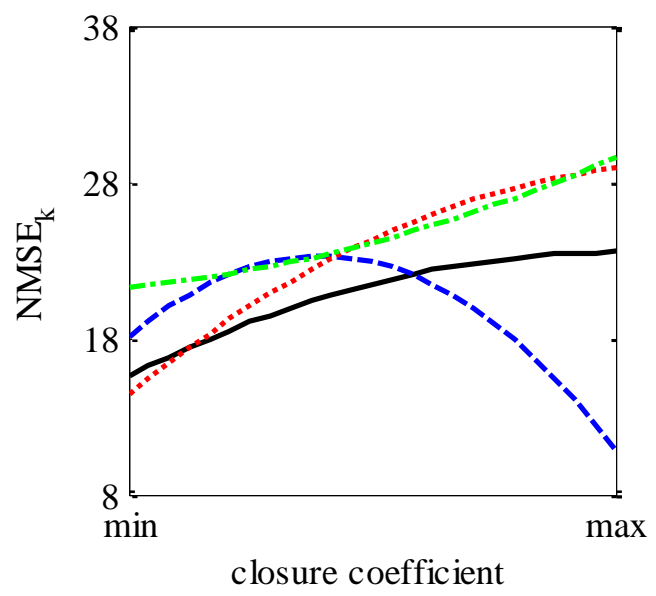

(d)

Figure 10 Variation of the validation metrics for the sheltered building scenario: (a) $\boldsymbol{F} A C 2_{U},(b) \boldsymbol{F} \boldsymbol{B}_{k}$, (c) $q_{U}$, (d) $N M S E_{k}$.

In Fig.10, variation of the validation metrics for streamwise velocity $U$ and turbulent kinetic energy $k$ is shown for the sheltered cross-ventilated building model. The highest $\mathrm{FAC}_{\mathrm{U}}$ is obtained for the higher values of $C_{\varepsilon 2}$ and lower values of $C_{\mu}$ in the considered ranges. Fractional bias for the turbulent kinetic energy $\mathrm{FB}_{\mathrm{k}}$ strongly depends on $C_{\varepsilon 2}$. The result shows a higher agreement with the experimental data for the higher values of $C_{\varepsilon 2} . N M S E_{k}$ is considerably high, but its value strongly depends on $C_{\varepsilon 2}$ and $C_{\mu}$. For low values of $C_{\mu}$ and high values of $C_{\varepsilon 2}, N M S E_{k}$ is in its minimum value. High value of hit rate parameter for the velocity $\left(q_{U}\right)$ is also estimated for the lower values of $C_{\varepsilon 1}$ and higher values of $C_{\varepsilon 2}$ and $C_{\mu}$. Thus, typical variation of the validation metrics for the sheltered building test case shows a strong effect of $C_{\varepsilon 2}$ on the CFD result accuracy although it reveals that the variation of $\sigma_{k}$ has a very low impact on the CFD results. Therefore, it was concluded that only $C_{\varepsilon 1}, C_{\varepsilon 2}, C_{\mu}$ and $\sigma_{\varepsilon}$ are required to be considered in the optimization process while the value of $\sigma_{k}$ was assumed as its default value of 1 . 


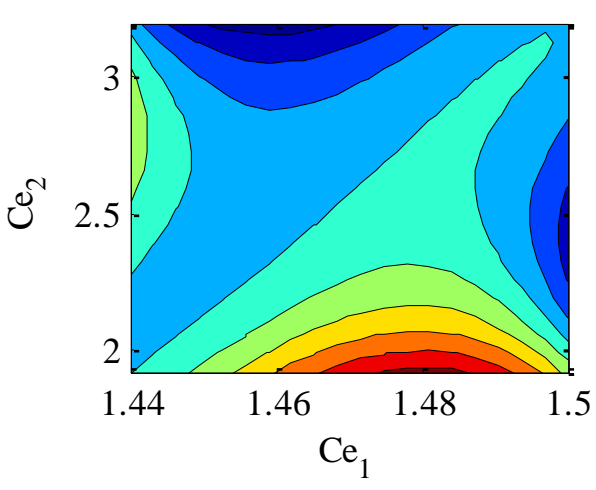

(a) mean $N M S E_{k}$

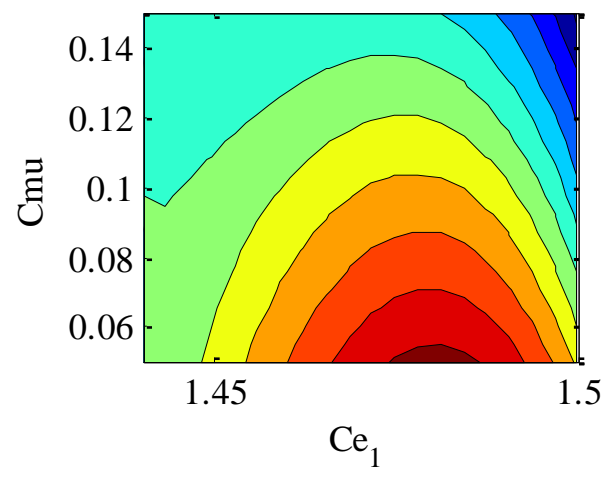

(c) mean $N M S E_{k}$

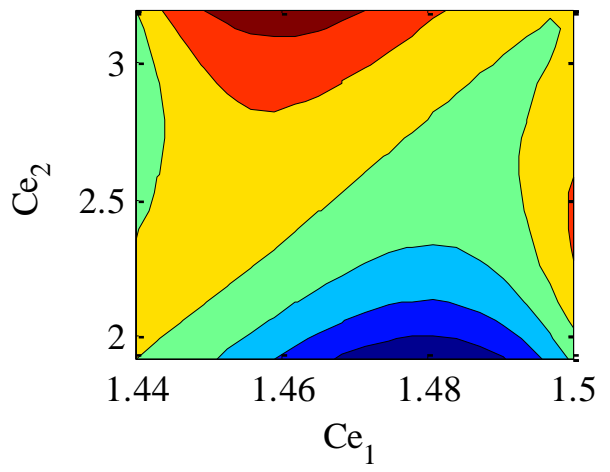

(b) mean $F A C 2_{U}$

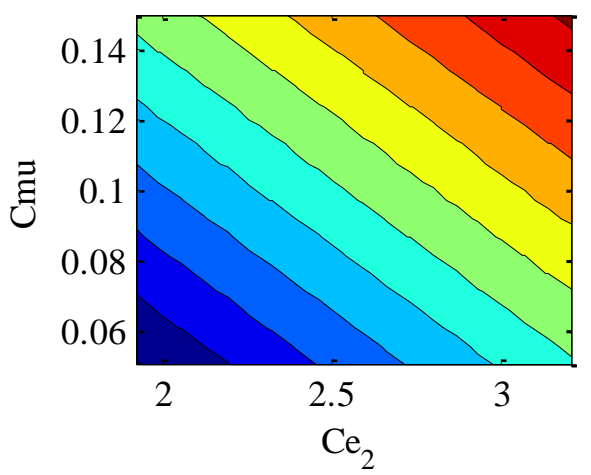

(d) mean $\mathrm{FAC2}_{U}$

\section{Min Max \\ Figure 11 Variation of the validation metrics for the optimization process of the sheltered building.}

As depicted in Fig. 11, contours of variation of the validation metrics during the optimization process are presented. These graphs determine the range of the closure coefficients in which higher agreement between the CFD and experimental results occurs with the highest probability. The modified value for $C_{\varepsilon 1}$ is close to its default value of 1.44 , however, values close to $C_{\varepsilon 1}=1.5$ show better accuracy in terms of validation metrics. The values of $C_{\varepsilon 2}$ between $2.7 \leq C_{\varepsilon 2} \leq 3.2$ show the highest agreement between the CFD and experimental data. In this case, the mean values of $N M S E_{k}$ and $F A C 2_{U}$ are around their minimum and maximum values, respectively. For $C_{\mu}$, a higher value than its default value (i.e. $C_{\mu}=0.09$ ) seems to result in a closer agreement with the experimental data. In terms of mean value of $N M S E_{k}$, a range of $0.11 \leq C_{\mu} \leq 0.14$ is preferable, but if the mean value of $F A C 2_{U}$ is considered, then values between $0.13 \leq C_{\mu} \leq 0.15$ show a better agreement. In Fig. 12, the standard deviation contours of the validation metrics related to the optimization process are furthermore displayed. Fig.12 (a) shows that the minimum standard deviation of $N M S E_{k}$ occurs for $C_{\varepsilon 1} \approx 1.5$ and $2.7 \leq C_{\varepsilon 2} \leq 3.2$. However, according to Fig.12 (b), in the mentioned ranges, the standard deviation value of $F A C 2_{U}$ is relatively high. 


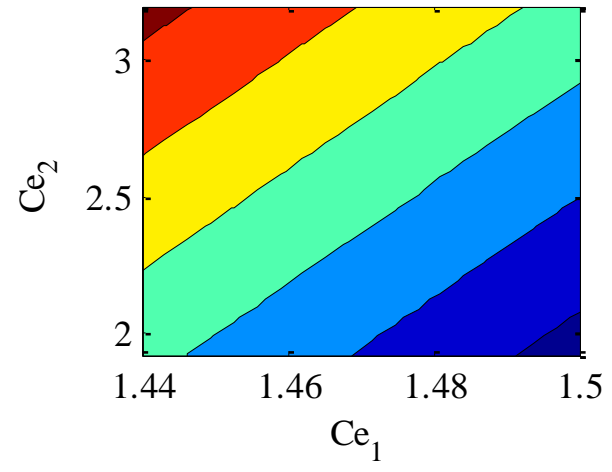

(a) standard deviation of $N M S E_{k}$

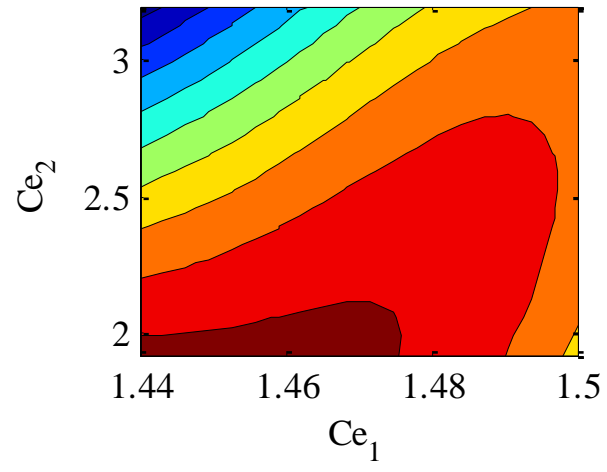

(b) standard deviation of $F A C 2_{U}$

\section{Min}

Figure 12 Contours of standard deviation of the validation metrics for the sheltered building: (a) NMSE $\boldsymbol{k}_{\boldsymbol{k}}$, (b) $F A C 2_{U}$.

As illustrated in Fig. 13, streamlines at the vertical center of the building model are shown for the standard $k-\varepsilon$ with default and modified closure coefficients models while they are compared with those of the experiment. As described before, in the case of the standard closure coefficients (see Fig.13(b)), the velocity field inside the building model is not accurately simulated mainly due to the poor prediction of the momentum diffusion and TKE inside the upstream and downstream cavities around the target building, and also incorrect pressure difference estimation across the openings of the target building. For the case of the modified coefficients, as shown in Fig.13 (c), the CFD model shows a considerable improvement in predicting the velocity field inside the target building. In this case, as shown in Fig. 8, pressure difference over the windward and leeward openings are noticeably increased. No wall pressure measurement was done in the experiment, however, an estimation of the pressure coefficient on building walls can be obtained using a method similar to Quan et al. [69] for low-rise sheltered buildings. The difference between the average pressure coefficent at the windward surface and the leeward surface $\left(\Delta C_{p}=C_{p_{\text {windward }}}-C_{p_{\text {leeward }}}\right)$ for a plan-area density $C_{A}=0.25$ was reported to be about $\Delta C_{p}=0.4$. The CFD result for $\Delta C_{p}$ for the standard $k-\varepsilon$ with default coefficients, $R N G k-\varepsilon$ and $S S T$ are $0.048,-0.037$ and -0.053 , respectively. For the case of the $k-\varepsilon$ model with modified coefficents $\Delta C_{p}=0.23$, which is noticably closer to the expected value reported in [69]. As a result, the incoming jet through the windward opening has enough momentum to infiltrate the building and moves out from the leeward opening in a similar pattern as it can be seen in the experiment. Moreover, results of the standard $k-\varepsilon$ with the modified closure coefficients show a better agreement with the experiment than those of the BSL RSM with the default closure coefficient (Fig. 7(d)). 


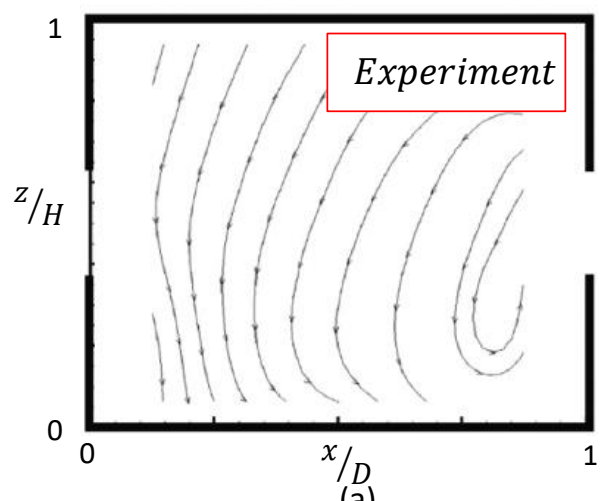

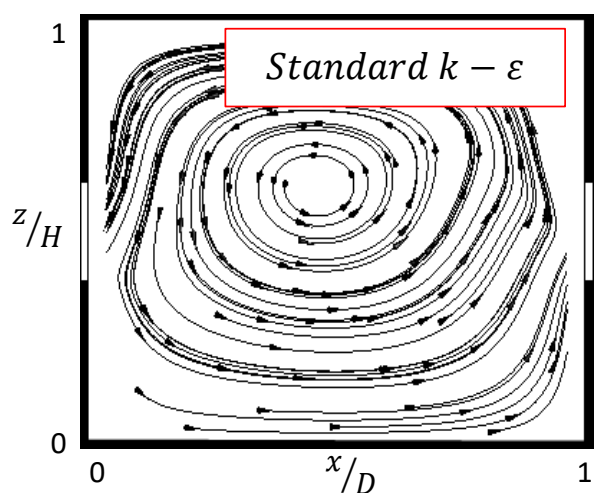

(b)

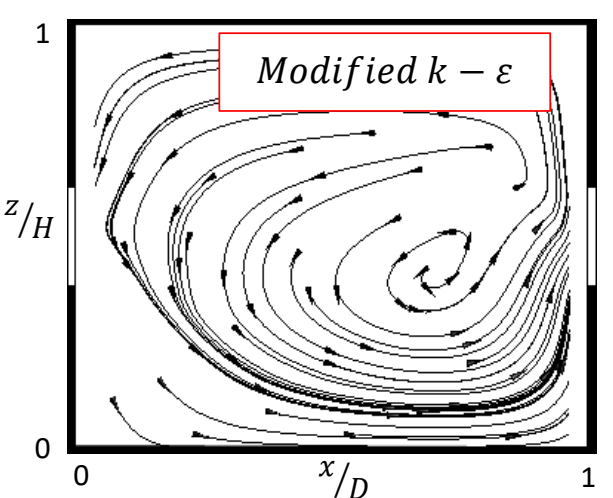

(c)

Figure 13 Streamline in the vertical center section of the sheltered building: (a) experiment by Tominaga and Blocken [1], (b) standard $\boldsymbol{k}-\boldsymbol{\varepsilon},(c) \boldsymbol{k}-\boldsymbol{\varepsilon}$ with the modified closure coefficients.

When the modified closure coefficients are used, not only the velocity field prediction accuracy increases, but the airflow rate calculation is also significantly improved. For the case of the modified closure coefficients, the non-dimensional airflow rate was estimated to be about 0.064 , which is a quite closer number to the experimental result with a less than $8 \%$ error. As shown in Fig.8, in the case of the modified coefficients, the pressure difference over the windward and leeward windows greatly rose in a way that airflow can pass through the windward opening and move out from the leeward opening. The highest pressure difference across the building and consequently the highest airflow rate were calculated for the standard $k-\varepsilon$ model using the optimized closure coefficients.

According to the results, average value of $k / U_{H}^{2}$ at the windward opening was increased from $2.2 \times 10^{-4}$ for the standard $k-\varepsilon$ (see Table 1) to $14.5 \times 10^{-4}$ when the modified coefficients were utilized, which resulted in a more accurate velocity gradient for the incoming jet crossing the opening. However, the value is still lower than that of the experiment. The reason is due to the inherent incapability of the steady RANS models to reproduce large-scale fluctuation inside the cavities around the buildings and inside the building model. As described in [1], the $k$ profile inside the building was high where the velocity gradient was high. In the case of the standard $k-\varepsilon$ with the modified coefficients, as depicted in Fig. 14 (a), the velocity gradient is modeled more accurately than the default coefficients results at the windward opening $\left(\frac{x}{D}=0.125\right)$. A greater production term is also 
estimated using the modified closure coefficient, and consequently a better velocity field and airflow rate were predicted for the sheltered building scenario. In Fig. 14 (b) and Fig. 14 (c), vertical profiles of the streamwise velocity are shown at $\frac{x}{D}=0.5$ and $\frac{x}{D}=0.875$. A good agreement between the CFD results of the modified closure coefficients and experiments can be also seen for the lower part of the sheltered building with an average error of less than $30 \%$. The streamwise velocity at the upper part of the building was under-predicted for both models which is related to the inaccuracy of models in predicting the TKE at that region.

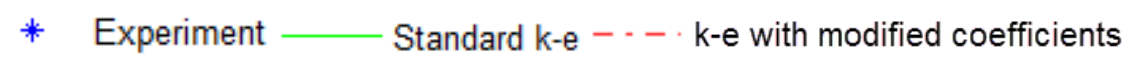

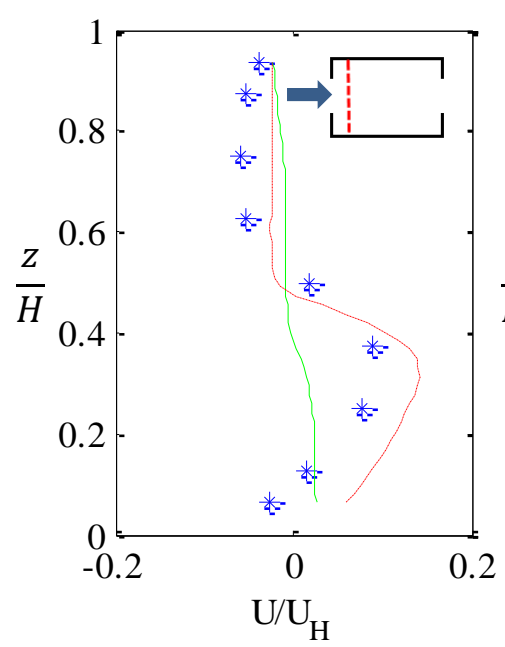

(a)

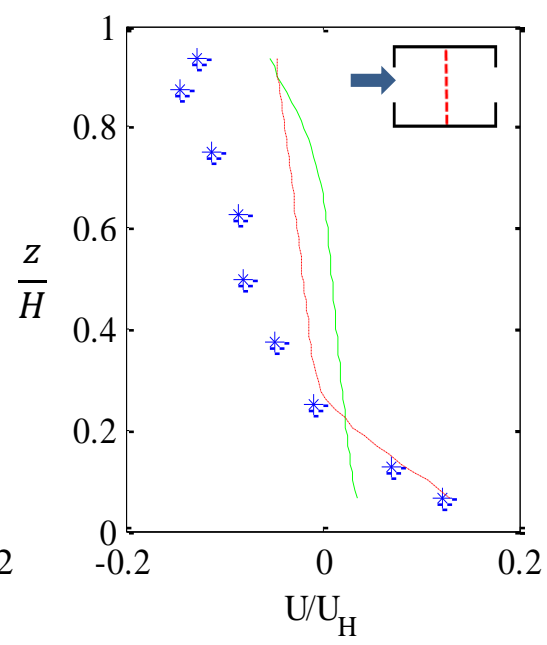

(b)

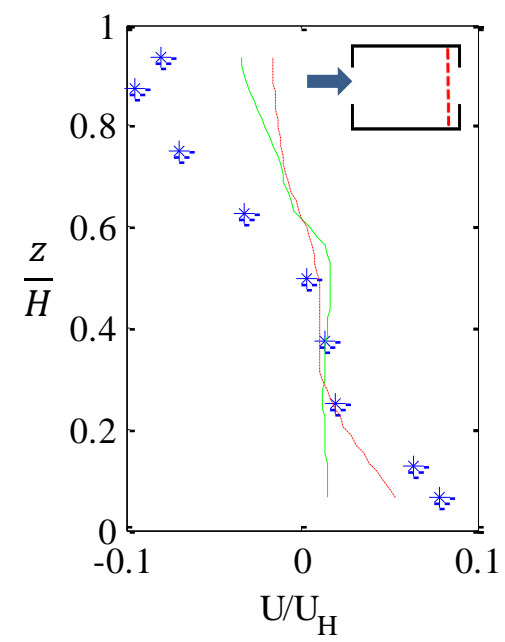

(c)

Figure 14 Vertical distribution of the streamwise velocity for the sheltered building at: (a) $\frac{x}{D}=\mathbf{0 . 1 2 5}$,

$$
\text { (b) } \frac{x}{D}=0.5,(c) \frac{x}{D}=0.875 \text {. }
$$

The effectiveness of the optimization methodology can be discussed in terms of validation metrics as they were considered as objective function for the optimizer. The hit rate value of the velocity field $\left(q_{U}\right)$ for the standard $k-\varepsilon$ model was 0.21 while it was predicted to be 0.35 for the $k-\varepsilon$ model with modified coefficients. The predicted values of $F A C 2$ for the velocity field were 0.12 and 0.29 using standard $k-\varepsilon$ with default and modified coefficients, respectively. The $N M S E$ values for TKE field showed a noticeable reduction from 49.4 to 9.8 but it is still far from the ideal value of 0 , which is mainly due to the inherent incapability of RANS models to capture the TKE inside the street canyons.

\section{Conclusions}

The accuracy of different RANS turbulence models were compared with the result of an experiment of a cross-ventilation for a sheltered building. As frequently stated in literature, a significant discrepancies between the experimental and CFD results can be observed for the distribution of streamwise velocity and TKE around the sheltered building. It was shown that, for the considered case study of a sheltered building, the RANS models not only fail to predict the crossing airflow through the target building, but they also fail to estimate the correct direction of flow inside the building. Using stochastic optimization and Monte Carlo Sampling technique, the coefficients of the standard $k-\varepsilon$ were modified in a way that the accuracy of the CFD model was considerably 
increased for a sheltered building test case. The following findings can be addressed as the main conclusion of the study:

- Application of RANS models for cross-ventilation modeling in densely packed urban areas is questionable due to their poor accuracy in reproducing the flow parameters in the wake regions inside the buildings and cavities around them.

- For the considered test case, all RANS models except the BSL RSM failed to predict velocity and TKE distributions inside the building and pressure difference between the windward and leeward openings.

- Airflow rate predictions for all RANS models, excluding BSL RSM, were incorrectly calculated in term of the direction from the leeward opening toward the windward one. However, the BSL RSM under-predicted the airflow rate by more than $70 \%$ error.

- The default value of the closure coefficients of the standard $k-\varepsilon$ model in commonly used CFD tools such as ANSYS CFX, ANSYS FLUENT, PHOENIX, and STAR-CMM+ are $C_{\mu}=0.09, C_{\varepsilon 1}=1.44, C_{\varepsilon 2}=1.92, \sigma_{k}=1$ and $\sigma_{\varepsilon}=1.3$, while the obtained values based on the optimization method for the considered sheltered building case are $1.45 \leq$ $C_{\varepsilon 1} \leq 1.5$, of $2.7 \leq C_{\varepsilon 2} \leq 3.2$ and $0.12 \leq C_{\mu} \leq 0.15$. The default value of $\sigma_{k}=1$ is suggested to be acceptable while the value of $\sigma_{\varepsilon}$ is obtained based on a correlation.

- For the $k-\varepsilon$ model with the optimized closure coefficients, prediction accuracy of the CFD model for the sheltered scenario was significantly improved for all the flow parameters, including the velocity and TKE distributions, and pressure coefficient. The crossing airflow rate prediction accuracy was also noticeably increased with an error about $8 \%$.

- Due to the inherent incapability of the steady RANS to reproduce large-scale turbulent fluctuations around and inside the building, TKE estimation inside the building is still lower than that of the experiment even for the optimized case.

It is important to note that the universality of the modified coefficient and their physical meanings for other flow fields are still remaining as a challenging issue. Therefore, future work will be focused to extend the proposed optimization approach in this study to other flows in urban areas. This will provide a set of appropriate closure coefficients for RANS models to achieve higher accuracy for design and analysis of ventilated buildings.

\section{Acknowledgment}

The authors would like to acknowledge the financial support from the University of Guilan.

\section{References}

[1] Y. Tominaga, B. Blocken, Wind tunnel experiments on cross-ventilation flow of a generic building with contaminant dispersion in unsheltered and sheltered conditions, Building and Environment, 92 (2015) 452-461.

[2] H.-x. Zhao, F. Magoulès, A review on the prediction of building energy consumption, Renewable and Sustainable Energy Reviews, 16(6) (2012) 3586-3592. 
[3] W. Goetzler, R. Zogg, J. Young, J. Schmidt, Energy Savings Potential and Research, Development, \& Demonstration Opportunities for Residential Building Heating, Ventilation, and Air Conditioning Systems, Navigant Consulting, Inc., Burlington, MA (United States), 2012.

[4] J. Ji, J.Y. Han, C.G. Fan, Z.H. Gao, J.H. Sun, Influence of cross-sectional area and aspect ratio of shaft on natural ventilation in urban road tunnel, International Journal of Heat and Mass Transfer, 67 (2013) 420-431.

[5] J. Serrano-Arellano, J. Xamán, G. Álvarez, Optimum ventilation based on the ventilation effectiveness for temperature and $\mathrm{CO} 2$ distribution in ventilated cavities, International Journal of Heat and Mass Transfer, 62 (2013) 9-21.

[6] W. Guo, X. Liu, X. Yuan, Study on Natural Ventilation Design Optimization Based on CFD Simulation for Green Buildings, Procedia Engineering, 121 (2015) 573-581.

[7] Q. Chen, Ventilation performance prediction for buildings: A method overview and recent applications, Building and environment, 44(4) (2009) 848-858.

[8] R. Ramponi, B. Blocken, CFD simulation of cross-ventilation for a generic isolated building: impact of computational parameters, Building and Environment, 53 (2012) 34-48.

[9] R. Teppner, B. Langensteiner, W. Meile, G. Brenn, S. Kerschbaumer, Air change rates driven by the flow around and through a building storey with fully open or tilted windows: An experimental and numerical study, Energy and Buildings, 80 (2014) 570-583.

[10] K. Visagavel, P. Srinivasan, Analysis of single side ventilated and cross ventilated rooms by varying the width of the window opening using CFD, Solar Energy, 83(1) (2009) 2-5.

[11] R. Evins, J. Allegrini, P. Moonen, Emulating site-specific wind flow information for use in building energy simulations, Building Simulation and Optimization (BSO 2014), London, UK, (2014).

[12] J. Liu, J. Srebric, N. Yu, Numerical simulation of convective heat transfer coefficients at the external surfaces of building arrays immersed in a turbulent boundary layer, International Journal of Heat and Mass Transfer, 61 (2013) 209-225.

[13] A. Nottrott, S. Onomura, A. Inagaki, M. Kanda, J. Kleissl, Convective heat transfer on leeward building walls in an urban environment: Measurements in an outdoor scale model, International Journal of Heat and Mass Transfer, 54(15-16) (2011) 3128-3138.

[14] S.-Y. Wu, Z.-G. Shen, L. Xiao, D.-L. Li, Experimental study on combined convective heat loss of a fully open cylindrical cavity under wind conditions, International Journal of Heat and Mass Transfer, 83 (2015) 509-521.

[15] J. Yam, Y. Li, Z. Zheng, Nonlinear coupling between thermal mass and natural ventilation in buildings, International Journal of Heat and Mass Transfer, 46(7) (2003) 1251-1264.

[16] P.A. Mirzaei, J. Carmeliet, Dynamical computational fluid dynamics modeling of the stochastic wind for application of urban studies, Building and Environment, 70 (2013) 161-170.

[17] A. Mochida, H. Yoshino, S. Miyauchi, T. Mitamura, Total analysis of cooling effects of crossventilation affected by microclimate around a building, Solar Energy, 80(4) (2006) 371-382.

[18] P.-Y. Cui, Z. Li, W.-Q. Tao, Investigation of Re-independence of turbulent flow and pollutant dispersion in urban street canyon using numerical wind tunnel (NWT) models, International Journal of Heat and Mass Transfer, 79 (2014) 176-188.

[19] P.-Y. Cui, Z. Li, W.-Q. Tao, Numerical investigations on Re-independence for the turbulent flow and pollutant dispersion under the urban boundary layer with some experimental validations, International Journal of Heat and Mass Transfer, 106 (2017) 422-436.

[20] J. Ji, Z.H. Gao, C.G. Fan, J.H. Sun, Large Eddy Simulation of stack effect on natural smoke exhausting effect in urban road tunnel fires, International Journal of Heat and Mass Transfer, 66 (2013) 531-542.

[21] M.R. Mokhtarzadeh-Dehghan, Numerical simulation and comparison with experiment of natural convection between two floors of a building model via a stairwell, International Journal of Heat and Mass Transfer, 54(1-3) (2011) 19-33. 
[22] Z. Tong, Y. Chen, A. Malkawi, Defining the Influence Region in neighborhood-scale CFD simulations for natural ventilation design, Applied Energy, 182 (2016) 625-633.

[23] W. Zhang, Q. Chen, Large eddy simulation of indoor airflow with a filtered dynamic subgrid scale model, International Journal of Heat and Mass Transfer, 43(17) (2000) 3219-3231.

[24] S.C. Saha, M.M.K. Khan, Y.T. Gu, Unsteady buoyancy driven flows and heat transfer through coupled thermal boundary layers in a partitioned triangular enclosure, International Journal of Heat and Mass Transfer, 68 (2014) 375-382.

[25] T. van Hooff, B. Blocken, Y. Tominaga, On the accuracy of CFD simulations of cross-ventilation flows for a generic isolated building: comparison of RANS, LES and experiments, Building and Environment, (2016).

[26] Y.C. Aydin, P.A. Mirzaei, Wind-driven ventilation improvement with plan typology alteration: A CFD case study of traditional Turkish architecture, in: Building Simulation, Springer, 2016, pp. 1-16.

[27] J.O. Cheung, C.-H. Liu, CFD simulations of natural ventilation behaviour in high-rise buildings in regular and staggered arrangements at various spacings, Energy and Buildings, 43(5) (2011) 1149-1158. [28] S. Cui, P. Stabat, D. Marchio, Numerical simulation of wind-driven natural ventilation: effects of loggia and facade porosity on air change rate, Building and Environment, (2016).

[29] N.R. Martins, G.C. da Graça, Validation of numerical simulation tools for wind-driven natural ventilation design, in: Building Simulation, Springer, 2016, pp. 75-87.

[30] R. Zhang, K.P. Lam, S.-C. Yao, Y. Zhang, Coupled EnergyPlus and computational fluid dynamics simulation for natural ventilation, Building and Environment, 68 (2013) 100-113.

[31] F. Haghighat, P.A. Mirzaei, Impact of non-uniform urban surface temperature on pollution dispersion in urban areas, in: Building Simulation, Springer, 2011, pp. 227-244.

[32] P.A. Mirzaei, F. Haghighat, A novel approach to enhance outdoor air quality: Pedestrian ventilation system, Building and Environment, 45(7) (2010) 1582-1593.

[33] P.A. Mirzaei, F. Haghighat, Pollution removal effectiveness of the pedestrian ventilation system, Journal of Wind Engineering and Industrial Aerodynamics, 99(1) (2011) 46-58.

[34] P.A. Mirzaei, F. Haghighat, A procedure to quantify the impact of mitigation techniques on the urban ventilation, Building and environment, 47 (2012) 410-420.

[35] Y. Tominaga, Flow around a high-rise building using steady and unsteady RANS CFD: Effect of largescale fluctuations on the velocity statistics, Journal of Wind Engineering and Industrial Aerodynamics, 142 (2015) 93-103.

[36] Y. Tominaga, A. Mochida, T. Shirasawa, R. Yoshie, H. Kataoka, K. Harimoto, T. Nozu, Cross comparisons of CFD results of wind environment at pedestrian level around a high-rise building and within a building complex, Journal of Asian architecture and building engineering, 3(1) (2004) 63-70.

[37] Y. Tominaga, T. Stathopoulos, Numerical simulation of dispersion around an isolated cubic building: model evaluation of RANS and LES, Building and Environment, 45(10) (2010) 2231-2239.

[38] R. Yoshie, A. Mochida, Y. Tominaga, H. Kataoka, K. Harimoto, T. Nozu, T. Shirasawa, Cooperative project for CFD prediction of pedestrian wind environment in the Architectural Institute of Japan, Journal of wind engineering and industrial aerodynamics, 95(9) (2007) 1551-1578.

[39] N. Kasim, S. Zaki, A. Hagishima, M. Ali, M. Shirakashi, N. Arai, A. Razak, CFD Study of Cross Ventilation Performance of Different Buildings Layouts, (2014).

[40] V. Yakhot, S.A. Orszag, Renormalization-group analysis of turbulence, Physical review letters, 57(14) (1986) 1722.

[41] T.-H. Shih, W.W. Liou, A. Shabbir, Z. Yang, J. Zhu, A new k- $\epsilon$ eddy viscosity model for high reynolds number turbulent flows, Computers \& Fluids, 24(3) (1995) 227-238.

[42] B.A. Younis, Y. Zhou, Accounting for mean-flow periodicity in turbulence closures, Physics of Fluids (1994-present), 18(1) (2006) 018102. 
[43] M. Lateb, R. Meroney, M. Yataghene, H. Fellouah, F. Saleh, M. Boufadel, On the use of numerical modelling for near-field pollutant dispersion in urban environments- A review, Environmental Pollution, 208 (2016) 271-283.

[44] S.B. Pope, Turbulent flows, in, IOP Publishing, 2001.

[45] W. Edeling, P. Cinnella, R.P. Dwight, H. Bijl, Bayesian estimates of parameter variability in the k- $\varepsilon$ turbulence model, Journal of Computational Physics, 258 (2014) 73-94.

[46] P. Weihing, B.A. Younis, B. Weigand, Heat transfer enhancement in a ribbed channel: Development of turbulence closures, International Journal of Heat and Mass Transfer, 76 (2014) 509-522.

[47] A. Shams, F. Roelofs, E. Baglietto, S. Lardeau, S. Kenjeres, Assessment and calibration of an algebraic turbulent heat flux model for low-Prandtl fluids, International Journal of Heat and Mass Transfer, 79

(2014) 589-601.

[48] P. Duynkerke, Application of the E- $\varepsilon$ turbulence closure model to the neutral and stable atmospheric boundary layer, Journal of the atmospheric sciences, 45(5) (1988) 865-880.

[49] H. Detering, D. Etling, Application of the E- $\varepsilon$ turbulence model to the atmospheric boundary layer, Boundary-Layer Meteorology, 33(2) (1985) 113-133.

[50] M.S. Mohamed, J.C. LaRue, The decay power law in grid-generated turbulence, Journal of Fluid Mechanics, 219 (1990) 195-214.

[51] S. Tavoularis, U. Karnik, Further experiments on the evolution of turbulent stresses and scales in uniformly sheared turbulence, Journal of Fluid Mechanics, 204 (1989) 457-478.

[52] M.C. Dunn, B. Shotorban, A. Frendi, Uncertainty quantification of turbulence model coefficients via Latin Hypercube Sampling method, Journal of Fluids Engineering, 133(4) (2011) 041402.

[53] S. Guillas, N. Glover, L. Malki-Epshtein, Bayesian calibration of the constants of the k- $\varepsilon$ turbulence model for a CFD model of street canyon flow, Computer Methods in Applied Mechanics and Engineering, 279 (2014) 536-553.

[54] Q.M.Z. Iqbal, A. Chan, Pedestrian level wind environment assessment around group of high-rise cross-shaped buildings: Effect of building shape, separation and orientation, Building and Environment, 101 (2016) 45-63.

[55] B.E. Launder, D. Spalding, The numerical computation of turbulent flows, Computer methods in applied mechanics and engineering, 3(2) (1974) 269-289.

[56] F.R. Menter, Two-equation eddy-viscosity turbulence models for engineering applications, AIAA journal, 32(8) (1994) 1598-1605.

[57] D. Choudhury, Introduction to the renormalization group method and turbulence modeling, Fluent Incorporated, 1993.

[58] V. Yakhot, S. Orszag, S. Thangam, T. Gatski, C. Speziale, Development of turbulence models for shear flows by a double expansion technique, Physics of Fluids A: Fluid Dynamics, 4(7) (1992) 1510-1520.

[59] D. Wilcox, Turbulence modeling for CFD. La Canada, California: DCW Industries, Inc, 5354 (1998)

124-128.

[60] A. CFX, Solver Theory Guide. Ansys, Inc., Canonsburg, PA, (2011).

[61] B. Launder, D. Spalding, Mathematical models of turbulence Academic Press, New York, (1972).

[62] P. Durbin, Separated flow computations with the k-epsilon-v-squared model, AIAA journal, 33(4) (1995) 659-664.

[63] B. Launder, B. Sharma, Application of the energy-dissipation model of turbulence to the calculation of flow near a spinning disc, Letters in heat and mass transfer, 1(2) (1974) 131-137.

[64] J. Kim, P. Moin, R. Moser, Turbulence statistics in fully developed channel flow at low Reynolds number, Journal of fluid mechanics, 177 (1987) 133-166.

[65] E.-S. Zanoun, F. Durst, H. Nagib, Evaluating the law of the wall in two-dimensional fully developed turbulent channel flows, Physics of Fluids (1994-present), 15(10) (2003) 3079-3089. 
[66] Y. Tominaga, A. Mochida, R. Yoshie, H. Kataoka, T. Nozu, M. Yoshikawa, T. Shirasawa, AlJ guidelines for practical applications of CFD to pedestrian wind environment around buildings, Journal of wind engineering and industrial aerodynamics, 96(10) (2008) 1749-1761.

[67] K. Schittkowski, NLPQLP: A Fortran implementation of a sequential quadratic programming algorithm with distributed and non-monotone line search-user's guide, in, Report, Department of Computer Science, University of Bayreuth, 2006.

[68] P. Koch, R.-J. Yang, L. Gu, Design for six sigma through robust optimization, Structural and Multidisciplinary Optimization, 26(3-4) (2004) 235-248.

[69] Y. Quan, Y. Tamura, M. Matsui, S. Cao, A. Yoshida, S. Xu, Interference effect of a surrounding building group on wind loads on flat roof of low-rise building: Part I, Distribution of local wind pressure coefficient, Wind Engineers, JAWE, 32 (2007) 211-212. 